\title{
Favorable pendant-amino metal chelation in VX nerve agent model systems
}

Indrajit Bandyopadhyay, Min-jeong Kim, Yoon Sup Lee ${ }^{*}$, and David G. Churchill ${ }^{*}$

Supporting Information

Department of Chemistry and School of Molecular Science (BK 21)

Korea Advanced Institute of Science and Technology

Daejeon, 305-701

Republic of Korea

yslee@mail.kaist.ac.kr: 82-42-869-2821

dchurchill@kaist.ac.kr: 82-42-869-2845

fax: 82-42-869-2810

26 January, 2006 
Table S1. ${ }^{13} \mathrm{C}$ and ${ }^{1} \mathrm{H}$ NMR isotropic chemical shifts (in ppm relative to TMS) and ${ }^{31} \mathrm{P}$ isotropic chemical shifts for the most stable protonated (1) and metalated complexes $(\mathbf{2}, \mathbf{5}, \mathbf{1 1}, \mathbf{1 9}, \mathbf{2 6}, \mathbf{2 8}, \mathbf{3 1})$ at GIAOB3LYP/6-31+G* $\mathrm{Be}^{+2}, \mathrm{Mg}^{2+}$ : Maximum downfield shift.

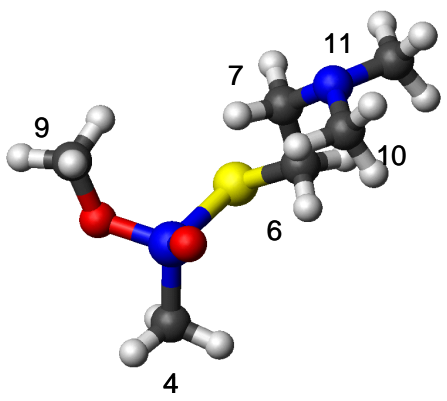

\begin{tabular}{|c|c|c|c|c|c|c|c|c|}
\hline Atom & 1 & 2 & 5 & 11 & 19 & 26 & 28 & 31 \\
\hline P1 & 54.0 & 147.7 & 67.7 & 65.5 & 58.4 & 86.3 & 83.6 & 74.6 \\
\hline C4 & 18.2 & 20.56 & 11.9 & 14.5 & 12.5 & 11.5 & 11.0 & 11.0 \\
\hline C6 & 25.3 & 54.0 & 27.4 & 27.9 & 27.4 & 36.7 & 32.3 & 29.3 \\
\hline C7 & 57.7 & 40.7 & 58.7 & 56.2 & 56.2 & 57.8 & 55.6 & 56.3 \\
\hline C9 & 43.8 & 40.7 & 51.8 & 47.3 & 51.1 & 63.5 & 60.0 & 57.2 \\
\hline C10 & 36.7 & 41.6 & 41.5 & 42.3 & 42.5 & 41.6 & 43.6 & 42.5 \\
\hline C11 & 36.8 & 35.5 & 37.1 & 37.5 & 37.3 & 40.6 & 39.4 & 37.3 \\
\hline H12 (C9) & 3.7 & 4.0 & 3.6 & 3.8 & 3.5 & 4.5 & 4.2 & 4.0 \\
\hline H13 (C9) & 3.5 & 4.3 & 3.7 & 3.4 & 3.6 & 4.5 & 4.3 & 4.1 \\
\hline H14 (C9) & 3.2 & 3.8 & 4.0 & 3.8 & 3.8 & 5.0 & 4.7 & 4.5 \\
\hline H15 (C4) & 1.1 & 1.9 & 1.6 & 1.5 & 1.6 & 2.1 & 2.0 & 1.9 \\
\hline H16 (C4) & 1.3 & 2.2 & 1.6 & 1.4 & 1.5 & 2.7 & 2.4 & 2.2 \\
\hline $\mathrm{H} 17$ (C4) & 1.2 & 2.0 & 1.5 & 1.7 & 1.4 & 2.3 & 2.1 & 1.9 \\
\hline $\mathrm{H18}$ (C6) & 2.3 & 3.4 & 2.8 & 3.0 & 2.8 & 4.0 & 3.8 & 3.5 \\
\hline H19 (C6) & 2.8 & 3.7 & 2.4 & 2.2 & 2.3 & 3.1 & 2.8 & 2.4 \\
\hline H20 (C7) & 3.0 & 2.6 & 2.6 & 2.4 & 2.4 & 3.2 & 2.8 & 2.6 \\
\hline H21 (C7) & 2.7 & 2.5 & 2.5 & 2.3 & 2.3 & 3.4 & 3.1 & 2.8 \\
\hline H22 (C11) & 2.3 & 2.3 & 2.2 & 2.2 & 2.2 & 3.1 & 3.0 & 2.8 \\
\hline H23 (C11) & 2.2 & 2.4 & 2.2 & 2.2 & 2.1 & 2.7 & 2.6 & 2.3 \\
\hline H24 (C11) & 2.2 & 2.3 & 2.2 & 2.2 & 2.0 & 2.8 & 2.8 & 2.4 \\
\hline H25 (C10) & 2.3 & 2.4 & 1.9 & 1.8 & 1.7 & 2.9 & 2.7 & 2.4 \\
\hline H26 (C10) & 2.1 & 2.3 & 2.1 & 2.1 & 1.9 & 2.9 & 2.7 & 2.3 \\
\hline H27 (C10) & 2.5 & 2.3 & 2.4 & 2.4 & 2.5 & 2.7 & 2.7 & 2.5 \\
\hline
\end{tabular}


Table S2. Bond lengths and bond length distortions in complexes (2-25) with respect to measurements of $\mathbf{1}$ (in parentheses).

\begin{tabular}{|c|c|c|c|c|c|}
\hline Metal ion & & $P-S$ & $P=O$ & P-OR & $C-S$ \\
\hline VX-Me & 1 & 2.133 & 1.500 & 1.627 & 1.854 \\
\hline $\mathbf{H}^{+}$ & 2 & $2.116(-0.017)$ & $1.515(0.015)$ & $1.613(-0.014)$ & $1.857(0.003)$ \\
\hline $\mathbf{H}^{+}$ & 3 & $2.071(-0.062)$ & $1.599(0.099)$ & $1.586(-0.041)$ & $1.875(0.021)$ \\
\hline $\mathbf{H}^{+}$ & 4 & $2.064(-0.069)$ & $1.481(-0.019)$ & $1.968(0.341)$ & $1.879(0.025)$ \\
\hline $\mathbf{L i}^{+}$ & 5 & $2.115(-0.018)$ & $1.515(0.015)$ & $1.596(-0.031)$ & $1.865(0.011)$ \\
\hline $\mathbf{L i}^{+}$ & 6 & $2.138(0.005)$ & $1.488(-0.012)$ & $1.681(0.054)$ & $1.866(0.012)$ \\
\hline $\mathbf{L i}^{+}$ & 7 & $2.084(-0.049)$ & $1.529(0.029)$ & $1.604(-0.023)$ & $1.864(0.01)$ \\
\hline $\mathbf{L i}^{+}$ & 8 & $2.110(-0.023)$ & $1.528(0.028)$ & $1.600(-0.027)$ & $1.856(0.002)$ \\
\hline $\mathbf{L i}^{+}$ & 9 & $2.145(0.012)$ & $1.693(0.193)$ & $1.484(-0.143)$ & $1.868(0.014)$ \\
\hline $\mathbf{L i}^{+}$ & 10 & $2.133(0.0)$ & $1.500(0.0)$ & $1.627(0.0)$ & $1.854(0.0)$ \\
\hline $\mathbf{N a}^{+}$ & 11 & $2.134(0.001)$ & $1.514(0.014)$ & $1.602(-0.025)$ & $1.868(0.014)$ \\
\hline $\mathrm{Na}^{+}$ & 12 & $2.137(0.004)$ & $1.490(-0.01)$ & $1.669(0.042)$ & $1.864(0.01)$ \\
\hline $\mathbf{N a}^{+}$ & 13 & $2.092(-0.041)$ & $1.521(0.021)$ & $1.610(-0.017)$ & $1.862(0.008)$ \\
\hline $\mathrm{Na}^{+}$ & 14 & $2.094(-0.039)$ & $1.516(0.016)$ & $1.608(-0.019)$ & $1.863(0.009)$ \\
\hline $\mathrm{Na}^{+}$ & 15 & $2.113(-0.02)$ & $1.521(0.021)$ & $1.604(-0.023)$ & $2.834(0.98)$ \\
\hline $\mathrm{Na}^{+}$ & 16 & $2.085(-0.048)$ & $1.513(0.013)$ & $1.661(0.034)$ & $1.860(0.006)$ \\
\hline $\mathrm{Na}^{+}$ & 17 & $2.189(0.056)$ & $1.488(-0.012)$ & $1.607(-0.02)$ & $1.854(0.0)$ \\
\hline $\mathrm{Na}^{+}$ & 18 & $2.140(0.007)$ & $1.487(-0.013)$ & $1.677(0.05)$ & $1.864(0.01)$ \\
\hline $\mathbf{K}^{+}$ & 19 & $2.124(-0.009)$ & $1.503(0.003)$ & $1.609(-0.018)$ & $1.864(0.01)$ \\
\hline $\mathbf{K}^{+}$ & 20 & $2.097(-0.036)$ & $1.513(0.013)$ & $1.614(-0.013)$ & $1.860(0.006)$ \\
\hline $\mathbf{K}^{+}$ & 21 & $2.131(-0.002)$ & $1.492(-0.008)$ & $1.658(0.031)$ & $1.863(0.009)$ \\
\hline $\mathbf{K}^{+}$ & 22 & $2.094(-0.039)$ & $1.509(0.009)$ & $1.650(0.023)$ & $1.857(0.003)$ \\
\hline $\mathbf{K}^{+}$ & 23 & $2.102(-0.031)$ & $1.516(0.016)$ & $1.610(-0.017)$ & $1.856(0.002)$ \\
\hline $\mathbf{K}^{+}$ & 24 & $2.107(-0.026)$ & $1.518(0.018)$ & $1.608(-0.019)$ & $1.857(0.003)$ \\
\hline $\mathbf{K}^{+}$ & 25 & $2.172(0.039)$ & $1.489(-0.011)$ & $1.614(-0.013)$ & $1.856(0.002)$ \\
\hline
\end{tabular}


Table S3. Bond lengths and bond length distortions in complexes (26-35) with respect to measurements of $\mathbf{1}$ (in parentheses).

\begin{tabular}{|c|c|c|c|c|c|}
\hline Metal ion & Geometry & P-S & $\mathbf{P}=\mathbf{O}$ & P-OR & C-S \\
\hline $\mathrm{Be}^{2+}$ & 26 & $2.187(0.054)$ & $1.567(0.067)$ & $1.552(-0.075)$ & $1.898(0.044)$ \\
\hline $\mathrm{Be}^{2+}$ & 27 & $2.204(0.071)$ & $1.473(-0.027)$ & $1.780(0.153)$ & $1.890(0.036)$ \\
\hline $\mathrm{Mg}^{2+}$ & 28 & $2.183(0.05)$ & $1.542(0.042)$ & $1.567(-0.06)$ & $1.890(0.036)$ \\
\hline $\mathbf{M g}^{2+}$ & 29 & $2.196(0.063)$ & $1.480(-0.02)$ & $1.732(0.105)$ & $1.884(0.03)$ \\
\hline $\mathbf{M g}^{2+}$ & 30 & $2.065(-0.068)$ & $1.551(0.051)$ & $1.684(0.057)$ & $1.876(0.022)$ \\
\hline $\mathbf{C a}^{2+}$ & 31 & $2.156(0.023)$ & $1.530(0.03)$ & $1.578(-0.049)$ & $1.875(0.021)$ \\
\hline $\mathbf{C a}^{2+}$ & 32 & $2.169(0.036)$ & $1.484(-0.016)$ & $1.710(0.083)$ & $1.876(0.022)$ \\
\hline $\mathbf{C a}^{2+}$ & 33 & $2.103(-0.03)$ & $1.555(0.055)$ & $1.579(-0.048)$ & $1.853(-0.001)$ \\
\hline $\mathbf{C a}^{2+}$ & 34 & $2.069(-0.064)$ & $1.535(0.035)$ & $1.694(0.067)$ & $1.865(0.011)$ \\
\hline $\mathrm{Ca}^{2+}$ & 35 & $2.135(0.002)$ & $1.546(0.046)$ & $1.575(-0.052)$ & $1.931(0.077)$ \\
\hline
\end{tabular}


Optimized Geometries:

\section{Geometry 1}

27 (No. of atoms)

\begin{tabular}{|c|c|c|c|c|c|c|c|}
\hline $1 \mathrm{P}$ & -1.732836 & -0.563493 & 0.188065 & 1 & 5 & 4 & 3 \\
\hline 20 & -0.814825 & -1.193541 & 1.187202 & 1 & 1 & & \\
\hline 30 & -2.784231 & 0.537573 & 0.761660 & 1 & 9 & 1 & \\
\hline $4 \mathrm{C}$ & -2.903442 & -1.685562 & -0.632004 & 1 & 17 & 16 & 15 \\
\hline $5 \mathrm{~S}$ & -0.678247 & 0.476740 & -1.346431 & 1 & 6 & 1 & \\
\hline $6 \mathrm{C}$ & 1.057887 & -0.089335 & -1.026940 & 1 & 19 & 18 & 7 \\
\hline $\mathrm{C}$ & 1.784801 & 0.800880 & 0.002051 & 1 & 21 & 20 & 8 \\
\hline $8 \mathrm{~N}$ & 3.182043 & 0.468064 & 0.260345 & 1 & 11 & 10 & 7 \\
\hline $9 \mathrm{C}$ & -2.331099 & 1.572888 & 1.655327 & 1 & 14 & 13 & 12 \\
\hline $10 \mathrm{C}$ & 4.085177 & 0.690824 & -0.858348 & 1 & 24 & 23 & 22 \\
\hline $11 \mathrm{C}$ & 3.394749 & -0.818100 & 0.911620 & 1 & 27 & 26 & 25 \\
\hline $12 \mathrm{H}$ & -1.845937 & 1.133517 & 2.531598 & 1 & 9 & & \\
\hline $13 \mathrm{H}$ & -1.638058 & 2.244828 & 1.137853 & 1 & 9 & & \\
\hline $14 \mathrm{H}$ & -3.224741 & 2.122427 & 1.955516 & 1 & 9 & & \\
\hline 15 & -2.339543 & -2.466147 & -1.150190 & 1 & 4 & & \\
\hline $\mathrm{H}$ & -3.541897 & -2.142876 & 0.130741 & 1 & 4 & & \\
\hline $17 \mathrm{H}$ & -3.522003 & -1.142188 & -1.351805 & 1 & 4 & & \\
\hline $\mathrm{H}$ & 1.538988 & -0.049085 & -2.009329 & 1 & 6 & & \\
\hline $\mathrm{H}$ & 1.024490 & -1.130399 & -0.699162 & 1 & 6 & & \\
\hline $\mathrm{H}$ & 1.242072 & 0.740216 & 0.951487 & 1 & 7 & & \\
\hline $\mathrm{H}$ & 1.742109 & 1.844058 & -0.336910 & 1 & 7 & & \\
\hline $\mathrm{H}$ & 3.985163 & -0.049783 & -1.676238 & 1 & 10 & & \\
\hline $\mathrm{H}$ & 3.915092 & 1.689051 & -1.278195 & 1 & 10 & & \\
\hline $\mathrm{H}$ & 5.121387 & 0.646648 & -0.501831 & 1 & 10 & & \\
\hline $\mathrm{H}$ & 3.259942 & -1.688061 & 0.240454 & 1 & 11 & & \\
\hline $\mathrm{H}$ & 4.418013 & -0.859223 & 1.304439 & 1 & 11 & & \\
\hline $27 \mathrm{H}$ & 2.699113 & -0.926712 & 1.749952 & 1 & 11 & & \\
\hline
\end{tabular}




\section{Geometry 2}

28

\begin{tabular}{|c|c|c|c|c|c|c|c|}
\hline $1 \mathrm{P}$ & 1.321539 & 0.047688 & -0.340198 & 1 & 11 & 4 & 3 \\
\hline 20 & 0.450090 & 1.256341 & -0.611862 & 1 & 28 & 1 & \\
\hline 30 & 1.973501 & 0.033980 & 1.134706 & 1 & 5 & 1 & \\
\hline $\mathrm{C}$ & 2.639670 & -0.207657 & -1.556690 & 1 & 15 & 14 & 13 \\
\hline $\mathrm{C}$ & 2.732616 & 1.167337 & 1.643835 & 1 & 24 & 17 & 16 \\
\hline 6 & -1.093558 & -1.494544 & 0.909709 & 1 & 19 & 18 & 11 \\
\hline $\mathrm{C}$ & -1.749058 & -0.118585 & 1.083146 & 1 & 21 & 20 & 8 \\
\hline $\mathrm{N}$ & -2.132531 & 0.612118 & -0.181452 & 1 & 28 & 10 & 9 \\
\hline $\mathrm{C}$ & -2.786930 & 1.922232 & 0.148889 & 1 & 27 & 22 & 12 \\
\hline 10 & -2.970094 & -0.194110 & -1.124198 & 1 & 26 & 25 & 23 \\
\hline $11 \mathrm{~S}$ & 0.210905 & -1.752625 & -0.386393 & 1 & 6 & 1 & \\
\hline 12 & -2.116786 & 2.497809 & 0.788957 & 1 & 9 & & \\
\hline 13 & 3.247031 & -1.079622 & -1.297718 & 1 & 4 & & \\
\hline 14 & 2.203444 & -0.336493 & -2.551650 & 1 & 4 & & \\
\hline 15 & 3.272274 & 0.686774 & -1.572926 & 1 & 4 & & \\
\hline $16 \mathrm{H}$ & 3.708049 & 1.212817 & 1.151461 & 1 & 5 & & \\
\hline 17 & 2.180410 & 2.098100 & 1.491431 & 1 & 5 & & \\
\hline 18 & -1.836346 & -2.256809 & 0.657347 & 1 & 6 & & \\
\hline 19 & -0.669785 & -1.765502 & 1.880455 & 1 & 6 & & \\
\hline $20 \mathrm{H}$ & -2.659536 & -0.241868 & 1.679727 & 1 & 7 & & \\
\hline 21 & -1.078725 & 0.558126 & 1.616103 & 1 & 7 & & \\
\hline 22 & -2.960551 & 2.467407 & -0.780219 & 1 & 9 & & \\
\hline 23 & -3.896914 & -0.482398 & -0.622241 & 1 & 10 & & \\
\hline 24 & 2.867492 & 0.974018 & 2.707812 & 1 & 5 & & \\
\hline 25 & -3.198343 & 0.420894 & -1.996404 & 1 & 10 & & \\
\hline 26 & -2.411778 & -1.075089 & -1.439750 & 1 & 10 & & \\
\hline 27 & -3.735034 & 1.734980 & 0.657714 & 1 & 9 & & \\
\hline $28 \mathrm{H}$ & -1.209361 & 0.858111 & -0.625565 & 1 & 2 & 8 & \\
\hline
\end{tabular}




\section{Geometry 3}

28

$\begin{array}{rrrrrrrrrr}1 & \mathrm{P} & 1.466296 & -0.289074 & -1.132802 & 1 & 11 & 4 & 3 & 2 \\ 2 & \mathrm{O} & 1.098281 & -1.295412 & -2.319141 & 1 & 28 & 1 & & \\ 3 & \mathrm{O} & 2.041352 & 1.001061 & -1.854972 & 1 & 5 & 1 & & \\ 4 & \mathrm{C} & 2.767750 & -1.192052 & -0.265944 & 1 & 15 & 14 & 13 & 1 \\ 5 & \mathrm{C} & 2.525106 & 2.227274 & -1.222702 & 1 & 24 & 17 & 16 & 3 \\ 6 & \mathrm{C} & -0.660488 & -1.242887 & 0.830324 & 1 & 19 & 18 & 11 & 7 \\ 7 & \mathrm{C} & -1.542224 & -0.792000 & 2.001508 & 1 & 21 & 20 & 8 & 6 \\ 8 & \mathrm{~N} & -2.446603 & 0.272066 & 1.586374 & 1 & 10 & 9 & 7 & \\ 9 & \mathrm{C} & -2.892515 & 1.109828 & 2.703975 & 1 & 27 & 22 & 12 & 8 \\ 10 & \mathrm{C} & -3.580371 & -0.208464 & 0.791523 & 1 & 26 & 25 & 23 & 8 \\ 11 & \mathrm{~S} & -0.160993 & 0.350606 & -0.023284 & 1 & 6 & 1 & & \\ 12 & \mathrm{H} & -2.025341 & 1.517633 & 3.232176 & 1 & 9 & & & \\ 13 & \mathrm{H} & 3.123284 & -0.610296 & 0.589452 & 1 & 4 & & & \\ 14 & \mathrm{H} & 2.397299 & -2.161263 & 0.079943 & 1 & 4 & & & \\ 15 & \mathrm{H} & 3.589703 & -1.360511 & -0.970309 & 1 & 4 & & & \\ 16 & \mathrm{H} & 1.747121 & 2.654183 & -0.586894 & 1 & 5 & & & \\ 17 & \mathrm{H} & 3.430168 & 2.012370 & -0.649970 & 1 & 5 & & & \\ 18 & \mathrm{H} & -1.194871 & -1.860046 & 0.105372 & 1 & 6 & & & \\ 19 & \mathrm{H} & 0.217876 & -1.784035 & 1.189473 & 1 & 6 & & & \\ 20 & \mathrm{H} & -2.064447 & -1.681269 & 2.401519 & 1 & 7 & & & \\ 21 & \mathrm{H} & -0.898905 & -0.403742 & 2.797289 & 1 & 7 & & & \\ 22 & \mathrm{H} & -3.478813 & 1.946375 & 2.313011 & 1 & 9 & & & \\ 23 & \mathrm{H} & -4.252171 & -0.862570 & 1.374781 & 1 & 10 & & & \\ 24 & \mathrm{H} & 2.752383 & 2.896908 & -2.050540 & 1 & 5 & & & \\ 25 & \mathrm{H} & -4.157362 & 0.647970 & 0.431549 & 1 & 10 & & & \\ 26 & \mathrm{H} & -3.232384 & -0.765188 & -0.084416 & 1 & 10 & & & \\ 27 & \mathrm{H} & -3.518294 & 0.556565 & 3.425720 & 1 & 9 & & & \\ 28 & \mathrm{H} & 0.451811 & -0.976521 & -2.977405 & 1 & 2 & & & \end{array}$




\section{Geometry 4}

28

$\begin{array}{rrrrrrrrrr}1 & \mathrm{P} & -1.706445 & -0.631640 & -0.160043 & 1 & 11 & 4 & 3 & 2 \\ 2 & \mathrm{O} & -2.322070 & -0.587077 & 1.186173 & 1 & 1 & & & \\ 3 & \mathrm{O} & -2.507762 & 0.942130 & -1.029353 & 1 & 28 & 5 & 1 & \\ 4 & \mathrm{C} & -2.313891 & -1.817914 & -1.383152 & 1 & 15 & 14 & 13 & 1 \\ 5 & \mathrm{C} & -3.465285 & 1.796990 & -0.287225 & 1 & 24 & 17 & 16 & 3 \\ 6 & \mathrm{C} & 0.774902 & 0.452996 & 1.175928 & 1 & 19 & 18 & 11 & 7 \\ 7 & \mathrm{C} & 2.207038 & -0.016760 & 1.435357 & 1 & 21 & 20 & 8 & 6 \\ 8 & \mathrm{~N} & 3.015407 & 0.108904 & 0.226427 & 1 & 10 & 9 & 7 & \\ 9 & \mathrm{C} & 4.147373 & -0.822726 & 0.200787 & 1 & 27 & 22 & 12 & 8 \\ 10 & \mathrm{C} & 3.449349 & 1.483452 & -0.037975 & 1 & 26 & 25 & 23 & 8 \\ 11 & \mathrm{~S} & 0.311585 & -0.328262 & -0.468927 & 1 & 6 & 1 & & \\ 12 & \mathrm{H} & 3.786601 & -1.848708 & 0.320305 & 1 & 9 & & & \\ 13 & \mathrm{H} & -1.886392 & -1.622630 & -2.371252 & 1 & 4 & & & \\ 14 & \mathrm{H} & -2.021659 & -2.820410 & -1.051442 & 1 & 4 & & & \\ 15 & \mathrm{H} & -3.405195 & -1.755369 & -1.425170 & 1 & 4 & & & \\ 16 & \mathrm{H} & -4.066683 & 2.313814 & -1.035154 & 1 & 5 & & & \\ 17 & \mathrm{H} & -4.068677 & 1.117251 & 0.310048 & 1 & 5 & & & \\ 18 & \mathrm{H} & 0.698536 & 1.535824 & 1.057435 & 1 & 6 & & & \\ 19 & \mathrm{H} & 0.077993 & 0.120280 & 1.948415 & 1 & 6 & & & \\ 20 & \mathrm{H} & 2.609715 & 0.555999 & 2.291641 & 1 & 7 & & & \\ 21 & \mathrm{H} & 2.185067 & -1.070661 & 1.729144 & 1 & 7 & & & \\ 22 & \mathrm{H} & 4.651804 & -0.748577 & -0.767012 & 1 & 9 & & & \\ 23 & \mathrm{H} & 4.139510 & 1.860981 & 0.737231 & 1 & 10 & & & \\ 24 & \mathrm{H} & -2.914123 & 2.487211 & 0.351786 & 1 & 5 & & & \\ 25 & \mathrm{H} & 3.960427 & 1.522137 & -1.004179 & 1 & 10 & & & \\ 26 & \mathrm{H} & 2.589052 & 2.158804 & -0.089795 & 1 & 10 & & & \\ 27 & \mathrm{H} & 4.886893 & -0.612998 & 0.993117 & 1 & 9 & & & \\ 28 & \mathrm{H} & -1.877665 & 1.474875 & -1.553481 & 1 & 3 & & & \\ & & & & & & & & \end{array}$




\section{Geometry 5}

28

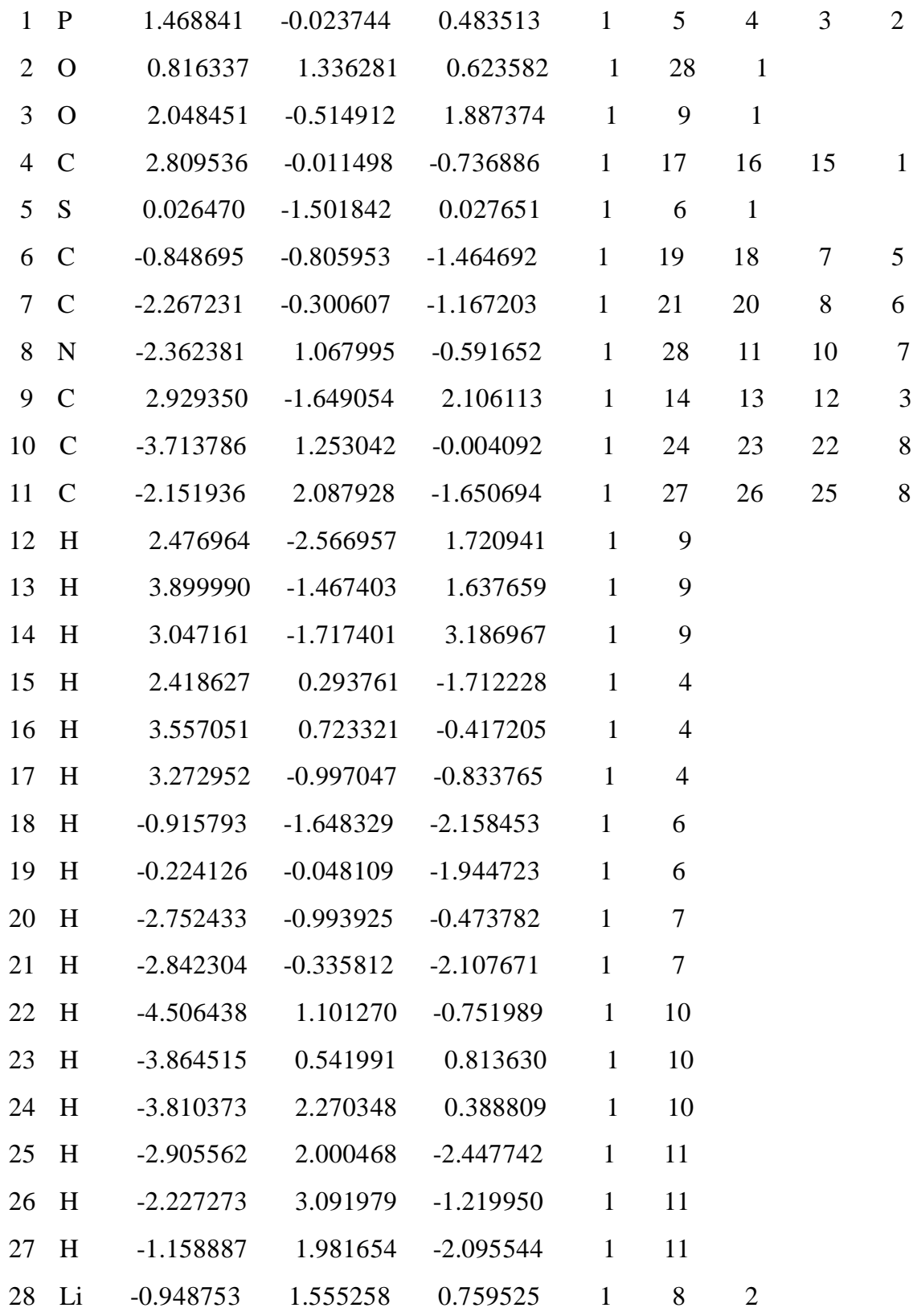




\section{Geometry 6}

28

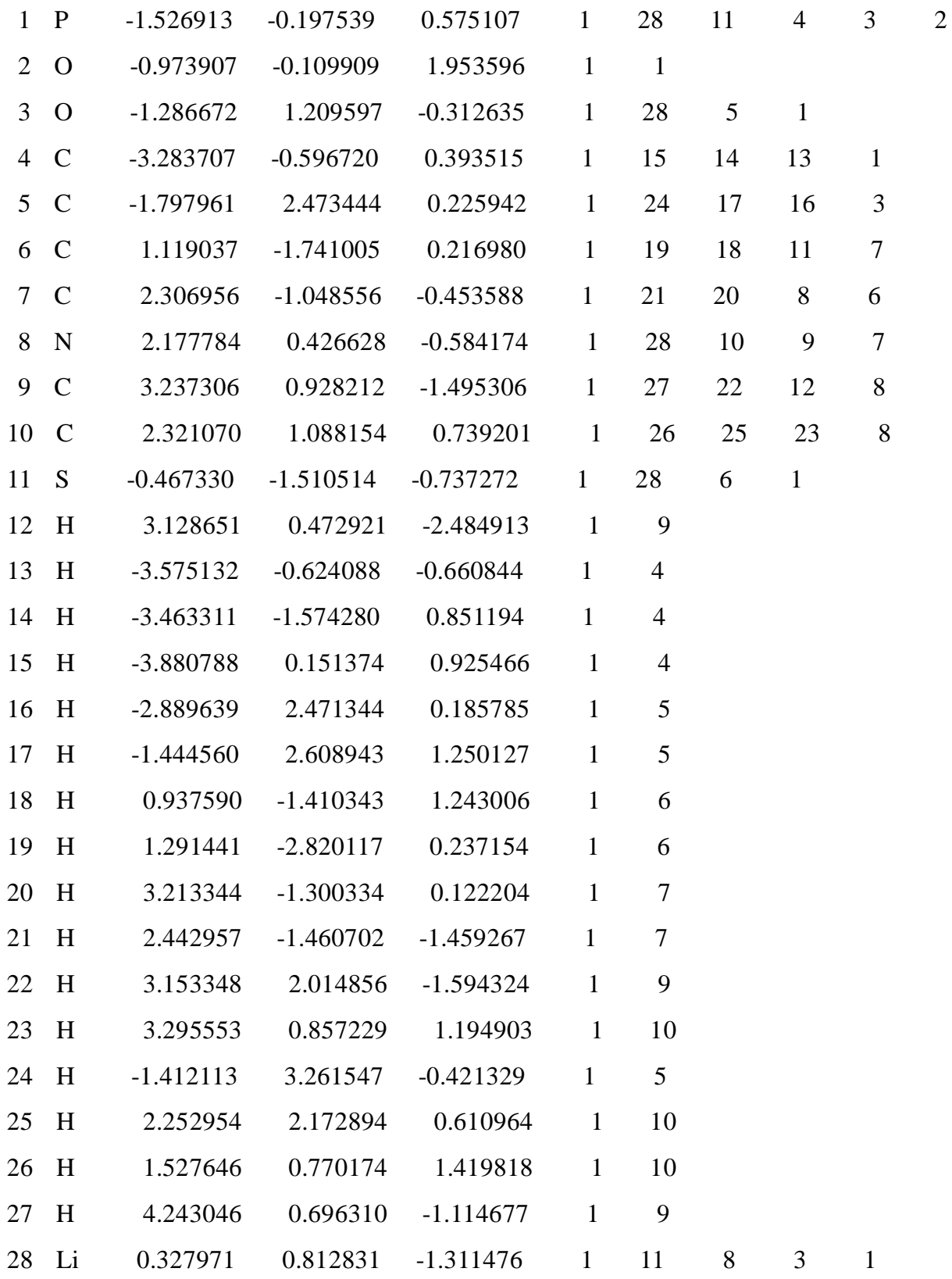




\section{Geometry 7}

28

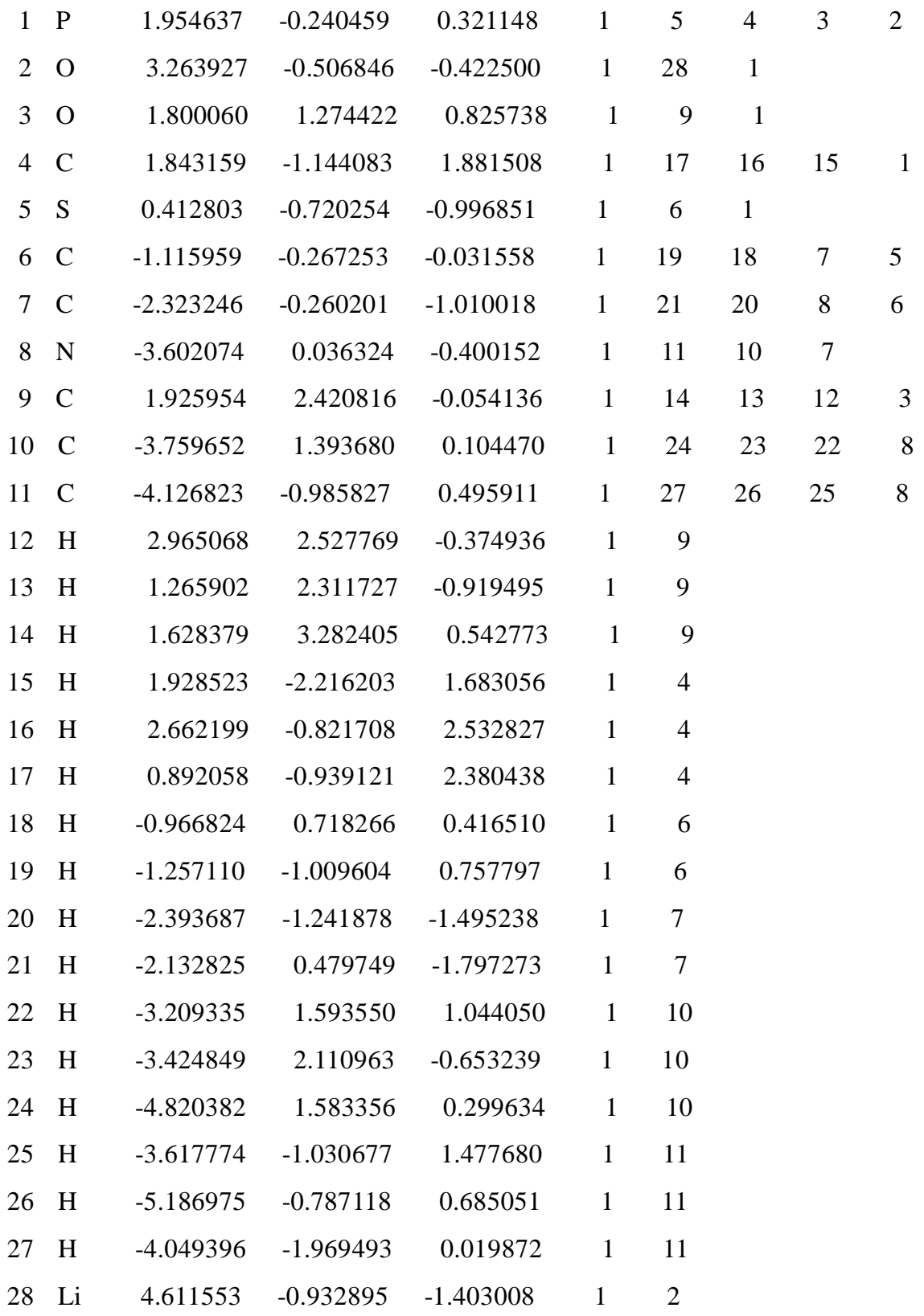




\section{Geometry 8}

28

\begin{tabular}{|c|c|c|c|c|c|c|c|c|}
\hline $1 \mathrm{P}$ & 1.704608 & 0.094360 & -0.422624 & 1 & 5 & 4 & 3 & 2 \\
\hline $2 \mathrm{O}$ & 0.776707 & 1.275986 & -0.699282 & 1 & 28 & 1 & & \\
\hline $\mathrm{O}$ & 2.841182 & 0.372198 & 0.663687 & 1 & 9 & 1 & & \\
\hline $4 \mathrm{C}$ & 2.689847 & -0.363657 & -1.863854 & 1 & 17 & 16 & 15 & 1 \\
\hline $5 \mathrm{~S}$ & 0.612180 & -1.568214 & 0.281132 & 1 & 6 & 1 & & \\
\hline $6 \mathrm{C}$ & -1.114930 & -1.117209 & -0.225515 & 1 & 19 & 18 & 7 & 5 \\
\hline $\mathrm{C}$ & -1.871064 & -0.314899 & 0.860860 & 1 & 21 & 20 & 8 & 6 \\
\hline $\mathrm{N}$ & -3.106314 & 0.322104 & 0.422853 & 1 & 11 & 10 & 7 & \\
\hline $9 \mathrm{C}$ & 2.588815 & 0.837611 & 2.015304 & 1 & 14 & 13 & 12 & 3 \\
\hline $10 \mathrm{C}$ & -4.150414 & -0.576152 & -0.068362 & 1 & 24 & 23 & 22 & 8 \\
\hline $11 \mathrm{C}$ & -2.951532 & 1.531415 & -0.342251 & 1 & 28 & 27 & 26 & 25 \\
\hline $12 \mathrm{H}$ & 2.229327 & 1.869077 & 1.987477 & 1 & 9 & & & \\
\hline $13 \mathrm{H}$ & 1.866330 & 0.184358 & 2.511604 & 1 & 9 & & & \\
\hline $14 \mathrm{H}$ & 3.551189 & 0.788558 & 2.523435 & 1 & 9 & & & \\
\hline $15 \mathrm{H}$ & 2.023880 & -0.640643 & -2.686185 & 1 & 4 & & & \\
\hline $\mathrm{H}$ & 3.305776 & 0.492040 & -2.159241 & 1 & 4 & & & \\
\hline $\mathrm{H}$ & 3.335932 & -1.211826 & -1.620708 & 1 & 4 & & & \\
\hline $\mathrm{H}$ & -1.596713 & -2.081298 & -0.411590 & 1 & 6 & & & \\
\hline $\mathrm{H}$ & -1.073561 & -0.591306 & -1.182960 & 1 & 6 & & & \\
\hline $\mathrm{H}$ & -1.215970 & 0.465164 & 1.267417 & 1 & 7 & & & \\
\hline $\mathrm{H}$ & -2.104851 & -0.986940 & 1.694757 & 1 & 7 & & & \\
\hline $\mathrm{H}$ & -3.945094 & -0.995873 & -1.068640 & 1 & 10 & & & \\
\hline $\mathrm{H}$ & -4.268735 & -1.404540 & 0.637016 & 1 & 10 & & & \\
\hline $\mathrm{H}$ & -5.101805 & -0.036927 & -0.114525 & 1 & 10 & & & \\
\hline $\mathrm{H}$ & -2.491055 & 1.393894 & -1.359167 & 1 & 11 & & & \\
\hline $\mathrm{H}$ & -3.926604 & 1.989525 & -0.532781 & 1 & 11 & & & \\
\hline $\mathrm{H}$ & -2.382209 & 2.269650 & 0.253636 & 1 & 11 & & & \\
\hline $\mathrm{Li}$ & -0.637693 & 2.250084 & -0.943537 & 1 & 11 & 2 & & \\
\hline
\end{tabular}




\section{Geometry 9}

28

\begin{tabular}{|c|c|c|c|c|c|c|c|c|}
\hline $1 \mathrm{P}$ & 1.576630 & -0.209042 & -0.895394 & 1 & 28 & 5 & 4 & 3 \\
\hline 20 & 0.794094 & 0.283227 & -2.102584 & 1 & 28 & 1 & & \\
\hline $\mathrm{O}$ & 1.897458 & 1.266592 & -0.189015 & 1 & 28 & 9 & 1 & \\
\hline $4 \mathrm{C}$ & 3.169148 & -0.999056 & -1.231056 & 1 & 17 & 16 & 15 & 1 \\
\hline $5 \mathrm{~S}$ & 0.585420 & -1.491220 & 0.397082 & 1 & 6 & 1 & & \\
\hline $6 \mathrm{C}$ & -1.192168 & -0.964208 & 0.203343 & 1 & 19 & 18 & 7 & 5 \\
\hline $\mathrm{C}$ & -1.584967 & 0.225039 & 1.109523 & 1 & 21 & 20 & 8 & 6 \\
\hline $\mathrm{N}$ & -2.986813 & 0.587634 & 1.087817 & 1 & 11 & 10 & 7 & \\
\hline $9 \mathrm{C}$ & 2.599017 & 1.453476 & 1.073001 & 1 & 14 & 13 & 12 & 3 \\
\hline $10 \mathrm{C}$ & -3.891899 & -0.369099 & 1.719380 & 1 & 24 & 23 & 22 & 8 \\
\hline $11 \mathrm{C}$ & -3.485208 & 1.108605 & -0.178122 & 1 & 27 & 26 & 25 & 8 \\
\hline $12 \mathrm{H}$ & 2.114373 & 0.863895 & 1.854989 & 1 & 9 & & & \\
\hline $13 \mathrm{H}$ & 3.647508 & 1.165599 & 0.961885 & 1 & 9 & & & \\
\hline $14 \mathrm{H}$ & 2.529544 & 2.515505 & 1.307490 & 1 & 9 & & & \\
\hline $15 \mathrm{H}$ & 2.999140 & -1.865795 & -1.877359 & 1 & 4 & & & \\
\hline $16 \mathrm{H}$ & 3.824165 & -0.288764 & -1.746139 & 1 & 4 & & & \\
\hline $17 \mathrm{H}$ & 3.646990 & -1.335909 & -0.305214 & 1 & 4 & & & \\
\hline $\mathrm{H}$ & -1.736171 & -1.873742 & 0.475427 & 1 & 6 & & & \\
\hline $19 \mathrm{H}$ & -1.367189 & -0.770347 & -0.857346 & 1 & 6 & & & \\
\hline $20 \mathrm{H}$ & -0.996474 & 1.107103 & 0.815004 & 1 & 7 & & & \\
\hline $21 \mathrm{H}$ & -1.305579 & -0.010563 & 2.143568 & 1 & 7 & & & \\
\hline $22 \mathrm{H}$ & -4.065381 & -1.283929 & 1.124040 & 1 & 10 & & & \\
\hline $23 \mathrm{H}$ & -3.495904 & -0.660413 & 2.698035 & 1 & 10 & & & \\
\hline $24 \mathrm{H}$ & -4.863087 & 0.110128 & 1.879560 & 1 & 10 & & & \\
\hline $25 \mathrm{H}$ & -3.600321 & 0.347005 & -0.971336 & 1 & 11 & & & \\
\hline $26 \mathrm{H}$ & -4.466232 & 1.566882 & -0.016205 & 1 & 11 & & & \\
\hline $27 \quad \mathrm{H}$ & -2.810311 & 1.892301 & -0.545897 & 1 & 11 & & & \\
\hline $\mathrm{Li}$ & 0.873497 & 2.091889 & -1.773746 & 1 & 3 & 2 & 1 & \\
\hline
\end{tabular}




\section{Geometry 10}

28

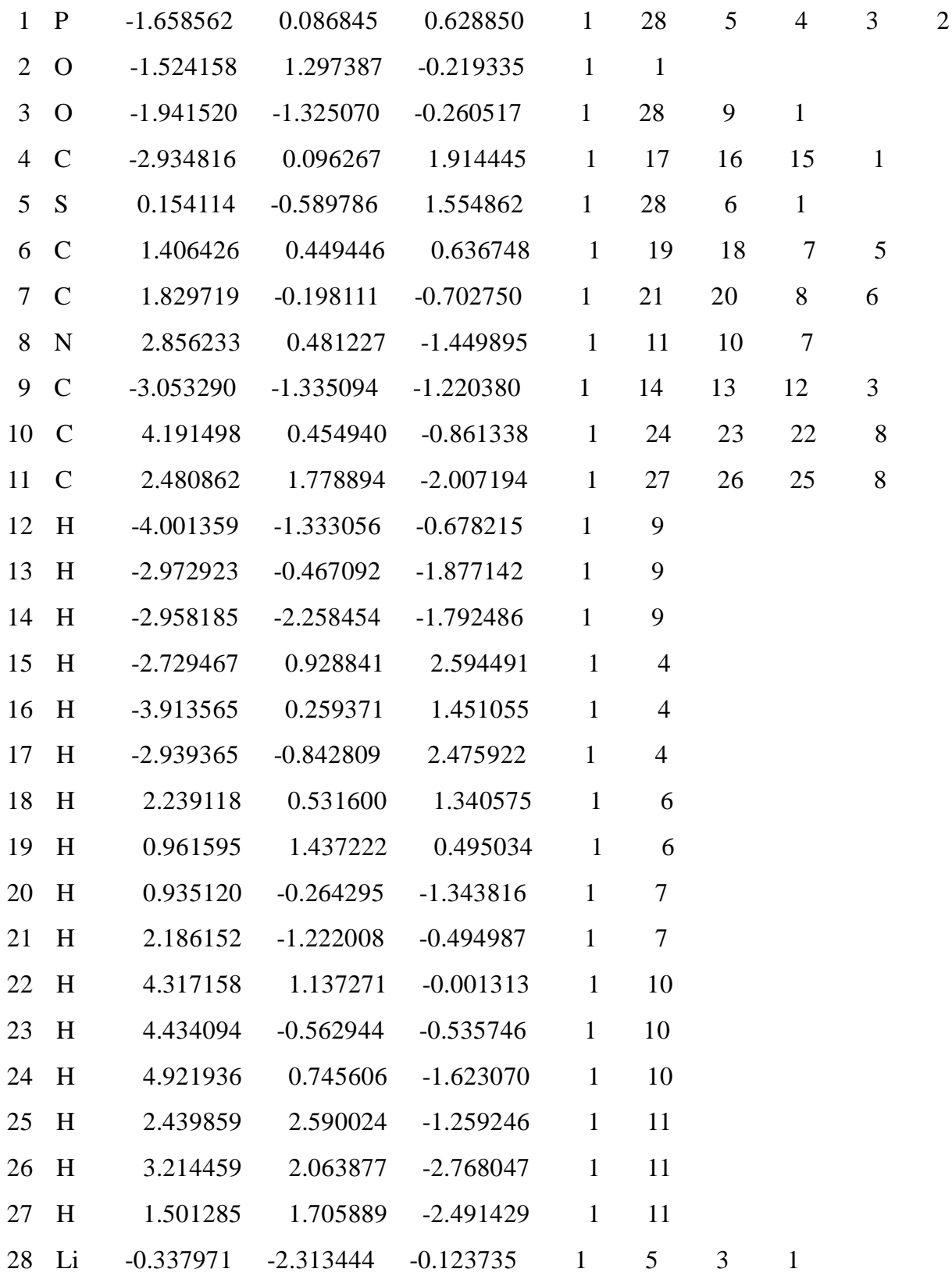




\section{Geometry 11}

28

$\begin{array}{rrrrrrrrrr}1 & \mathrm{P} & -1.732098 & -0.214414 & 0.387331 & 1 & 11 & 4 & 3 & 2 \\ 2 & \mathrm{O} & -1.030954 & 0.787986 & 1.279814 & 1 & 28 & 1 & & \\ 3 & \mathrm{O} & -3.070878 & 0.302332 & -0.324170 & 1 & 5 & 1 & & \\ 4 & \mathrm{C} & -2.298900 & -1.713780 & 1.222862 & 1 & 15 & 14 & 13 & 1 \\ 5 & \mathrm{C} & -3.261282 & 1.656530 & -0.805387 & 1 & 24 & 17 & 16 & 3 \\ 6 & \mathrm{C} & 0.963905 & -1.580868 & -0.484608 & 1 & 19 & 18 & 11 & 7 \\ 7 & \mathrm{C} & 2.316001 & -0.925582 & -0.794440 & 1 & 21 & 20 & 8 & 6 \\ 8 & \mathrm{~N} & 2.660465 & 0.244348 & 0.046528 & 1 & 28 & 10 & 9 & 7 \\ 9 & \mathrm{C} & 3.760902 & 1.000767 & -0.593043 & 1 & 27 & 22 & 12 & 8 \\ 10 & \mathrm{C} & 3.091067 & -0.191504 & 1.394208 & 1 & 26 & 25 & 23 & 8 \\ 11 & \mathrm{~S} & -0.482265 & -0.699493 & -1.272805 & 1 & 6 & 1 & & \\ 12 & \mathrm{H} & 3.439616 & 1.374284 & -1.570517 & 1 & 9 & & & \\ 13 & \mathrm{H} & -2.847680 & -2.349201 & 0.522685 & 1 & 4 & & & \\ 14 & \mathrm{H} & -1.450331 & -2.264845 & 1.638110 & 1 & 4 & & & \\ 15 & \mathrm{H} & -2.959883 & -1.411652 & 2.042540 & 1 & 4 & & & \\ 16 & \mathrm{H} & -3.018996 & 2.374716 & -0.018069 & 1 & 5 & & & \\ 17 & \mathrm{H} & -2.650387 & 1.827919 & -1.696795 & 1 & 5 & & & \\ 18 & \mathrm{H} & 0.797297 & -1.682897 & 0.591002 & 1 & 6 & & & \\ 19 & \mathrm{H} & 0.951651 & -2.587378 & -0.910824 & 1 & 6 & & & \\ 20 & \mathrm{H} & 3.095967 & -1.699975 & -0.688598 & 1 & 7 & & & \\ 21 & \mathrm{H} & 2.329589 & -0.609795 & -1.842646 & 1 & 7 & & & \\ 22 & \mathrm{H} & 4.039412 & 1.852849 & 0.035879 & 1 & 9 & & & \\ 23 & \mathrm{H} & 3.975247 & -0.847557 & 1.346870 & 1 & 10 & & & \\ 24 & \mathrm{H} & -4.317630 & 1.726353 & -1.064085 & 1 & 5 & & & \\ 25 & \mathrm{H} & 3.350328 & 0.682795 & 2.000641 & 1 & 10 & & & \\ 26 & \mathrm{H} & 2.287393 & -0.731875 & 1.902892 & 1 & 10 & & & \\ 27 & \mathrm{H} & 4.658965 & 0.378759 & -0.736454 & 1 & 9 & & & \\ 28 & \mathrm{Na} & 0.798036 & 1.679023 & 0.490353 & 1 & 8 & 2 & & \\ & & & & & & & & \end{array}$




\section{Geometry 12}

28

$\begin{array}{rlrrrrrrrr}1 & \mathrm{P} & -1.673377 & -0.296797 & 0.294488 & 1 & 11 & 4 & 3 & 2 \\ 2 & \mathrm{O} & -1.303683 & -0.280972 & 1.738287 & 1 & 1 & & & \\ 3 & \mathrm{O} & -1.531701 & 1.195824 & -0.438592 & 1 & 28 & 5 & 1 & \\ 4 & \mathrm{C} & -3.334311 & -0.894878 & -0.121168 & 1 & 15 & 14 & 13 & 1 \\ 5 & \mathrm{C} & -2.366491 & 2.287494 & 0.062614 & 1 & 24 & 17 & 16 & 3 \\ 6 & \mathrm{C} & 1.018642 & -1.803199 & 0.297819 & 1 & 19 & 18 & 11 & 7 \\ 7 & \mathrm{C} & 2.365116 & -1.137319 & 0.005588 & 1 & 21 & 20 & 8 & 6 \\ 8 & \mathrm{~N} & 2.392807 & 0.339101 & 0.106091 & 1 & 28 & 10 & 9 & 7 \\ 9 & \mathrm{C} & 3.706335 & 0.827226 & -0.370224 & 1 & 27 & 22 & 12 & 8 \\ 10 & \mathrm{C} & 2.182793 & 0.784923 & 1.503964 & 1 & 26 & 25 & 23 & 8 \\ 11 & \mathrm{~S} & -0.309360 & -1.382697 & -0.940554 & 1 & 28 & 6 & 1 & \\ 12 & \mathrm{H} & 3.866254 & 0.526134 & -1.411084 & 1 & 9 & & & \\ 13 & \mathrm{H} & -3.507241 & -0.862712 & -1.201353 & 1 & 4 & & & \\ 14 & \mathrm{H} & -3.425642 & -1.926092 & 0.232886 & 1 & 4 & & & \\ 15 & \mathrm{H} & -4.082299 & -0.283693 & 0.394471 & 1 & 4 & & & \\ 16 & \mathrm{H} & -3.406945 & 2.119435 & -0.226082 & 1 & 5 & & & \\ 17 & \mathrm{H} & -2.269990 & 2.365551 & 1.148106 & 1 & 5 & & & \\ 18 & \mathrm{H} & 0.625731 & -1.566331 & 1.289468 & 1 & 6 & & & \\ 19 & \mathrm{H} & 1.139219 & -2.887515 & 0.232186 & 1 & 6 & & & \\ 20 & \mathrm{H} & 3.096460 & -1.577642 & 0.707484 & 1 & 7 & & & \\ 21 & \mathrm{H} & 2.694450 & -1.410309 & -1.003481 & 1 & 7 & & & \\ 22 & \mathrm{H} & 3.738890 & 1.919916 & -0.309496 & 1 & 9 & & & \\ 23 & \mathrm{H} & 2.939175 & 0.356802 & 2.181555 & 1 & 10 & & & \\ 24 & \mathrm{H} & -2.002337 & 3.197772 & -0.415375 & 1 & 5 & & & \\ 25 & \mathrm{H} & 2.265902 & 1.875578 & 1.552151 & 1 & 10 & & & \\ 26 & \mathrm{H} & 1.189507 & 0.500553 & 1.859666 & 1 & 10 & & & \\ 27 & \mathrm{H} & 4.537324 & 0.429536 & 0.233901 & 1 & 9 & & & \\ 28 & \mathrm{Na} & 0.650262 & 1.247283 & -1.276838 & 1 & 11 & 8 & 3 & \end{array}$




\section{Geometry 13}

28

$\begin{array}{rrrrrrrrrr}1 & \mathrm{P} & 1.442020 & 0.111830 & 0.866274 & 1 & 5 & 4 & 3 & 2 \\ 2 & \mathrm{O} & 2.430589 & 1.216077 & 1.207791 & 1 & 28 & 1 & & \\ 3 & \mathrm{O} & 0.523321 & -0.357588 & 2.101920 & 1 & 9 & 1 & & \\ 4 & \mathrm{C} & 2.230684 & -1.454432 & 0.419620 & 1 & 17 & 16 & 15 & 1 \\ 5 & \mathrm{~S} & 0.223694 & 0.835724 & -0.672246 & 1 & 6 & 1 & & \\ 6 & \mathrm{C} & -0.980026 & -0.558903 & -0.944903 & 1 & 19 & 18 & 7 & 5 \\ 7 & \mathrm{C} & -2.160794 & -0.024843 & -1.800090 & 1 & 21 & 20 & 8 & 6 \\ 8 & \mathrm{~N} & -3.166579 & -1.009752 & -2.143621 & 1 & 11 & 10 & 7 & \\ 9 & \mathrm{C} & -0.238315 & 0.576431 & 2.903820 & 1 & 14 & 13 & 12 & 3 \\ 10 & \mathrm{C} & -3.943725 & -1.528529 & -1.026236 & 1 & 24 & 23 & 22 & 8 \\ 11 & \mathrm{C} & -2.743588 & -2.037836 & -3.085696 & 1 & 27 & 26 & 25 & 8 \\ 12 & \mathrm{H} & 0.438935 & 1.228339 & 3.461576 & 1 & 9 & & & \\ 13 & \mathrm{H} & -0.903393 & 1.170156 & 2.269376 & 1 & 9 & & & \\ 14 & \mathrm{H} & -0.821241 & -0.033828 & 3.593357 & 1 & 9 & & & \\ 15 & \mathrm{H} & 2.865548 & -1.301873 & -0.457752 & 1 & 4 & & & \\ 16 & \mathrm{H} & 2.841467 & -1.791808 & 1.263287 & 1 & 4 & & & \\ 17 & \mathrm{H} & 1.479642 & -2.217792 & 0.198813 & 1 & 4 & & & \\ 18 & \mathrm{H} & -1.327740 & -0.914973 & 0.028097 & 1 & 6 & & & \\ 19 & \mathrm{H} & -0.458716 & -1.367819 & -1.462897 & 1 & 6 & & & \\ 20 & \mathrm{H} & -1.763700 & 0.396447 & -2.732330 & 1 & 7 & & & \\ 21 & \mathrm{H} & -2.647797 & 0.793614 & -1.255346 & 1 & 7 & & & \\ 22 & \mathrm{H} & -3.390670 & -2.239311 & -0.382212 & 1 & 10 & & & \\ 23 & \mathrm{H} & -4.295104 & -0.698034 & -0.403547 & 1 & 10 & & & \\ 24 & \mathrm{H} & -4.823612 & -2.053077 & -1.413459 & 1 & 10 & & & \\ 25 & \mathrm{H} & -2.068649 & -2.799920 & -2.650604 & 1 & 11 & & & \\ 26 & \mathrm{H} & -3.627232 & -2.561418 & -3.465727 & 1 & 11 & & & \\ 27 & \mathrm{H} & -2.236348 & -1.572282 & -3.937989 & 1 & 11 & & & \\ 28 & \mathrm{Na} & 3.734414 & 2.843962 & 1.348887 & 1 & 2 & & & \end{array}$




\section{Geometry 14}

28

$\begin{array}{rlrrrrrrrr}1 & \mathrm{P} & -1.743073 & -0.134237 & 0.273218 & 1 & 11 & 4 & 3 & 2 \\ 2 & \mathrm{O} & -1.909055 & 1.366303 & 0.407989 & 1 & 28 & 1 & & \\ 3 & \mathrm{O} & -2.992000 & -0.715275 & -0.556908 & 1 & 5 & 1 & & \\ 4 & \mathrm{C} & -1.711605 & -0.940744 & 1.900706 & 1 & 15 & 14 & 13 & 1 \\ 5 & \mathrm{C} & -3.260762 & -2.120201 & -0.796945 & 1 & 24 & 17 & 16 & 3 \\ 6 & \mathrm{C} & 1.315518 & -0.088665 & 0.191427 & 1 & 19 & 18 & 11 & 7 \\ 7 & \mathrm{C} & 2.610531 & -0.156815 & -0.634297 & 1 & 21 & 20 & 8 & 6 \\ 8 & \mathrm{~N} & 3.740448 & 0.311749 & 0.159774 & 1 & 10 & 9 & 7 & \\ 9 & \mathrm{C} & 4.809649 & 0.862423 & -0.675129 & 1 & 27 & 22 & 12 & 8 \\ 10 & \mathrm{C} & 4.260409 & -0.711010 & 1.067716 & 1 & 26 & 25 & 23 & 8 \\ 11 & \mathrm{~S} & -0.084199 & -0.719826 & -0.863380 & 1 & 6 & 1 & & \\ 12 & \mathrm{H} & 4.420212 & 1.681125 & -1.288795 & 1 & 9 & & & \\ 13 & \mathrm{H} & -1.596350 & -2.024101 & 1.805562 & 1 & 4 & & & \\ 14 & \mathrm{H} & -0.879916 & -0.544992 & 2.491161 & 1 & 4 & & & \\ 15 & \mathrm{H} & -2.648046 & -0.712238 & 2.421104 & 1 & 4 & & & \\ 16 & \mathrm{H} & -2.392197 & -2.609214 & -1.245990 & 1 & 5 & & & \\ 17 & \mathrm{H} & -3.540547 & -2.615574 & 0.137047 & 1 & 5 & & & \\ 18 & \mathrm{H} & 1.116896 & 0.946480 & 0.477676 & 1 & 6 & & & \\ 19 & \mathrm{H} & 1.384950 & -0.708458 & 1.088038 & 1 & 6 & & & \\ 20 & \mathrm{H} & 2.762927 & -1.183699 & -1.019846 & 1 & 7 & & & \\ 21 & \mathrm{H} & 2.500462 & 0.498478 & -1.504646 & 1 & 7 & & & \\ 22 & \mathrm{H} & 5.597736 & 1.266370 & -0.032256 & 1 & 9 & & & \\ 23 & \mathrm{H} & 4.686650 & -1.578359 & 0.528292 & 1 & 10 & & & \\ 24 & \mathrm{H} & -4.100119 & -2.142721 & -1.491653 & 1 & 5 & & & \\ 25 & \mathrm{H} & 5.045261 & -0.275615 & 1.693383 & 1 & 10 & & & \\ 26 & \mathrm{H} & 3.472753 & -1.075422 & 1.734566 & 1 & 10 & & & \\ 27 & \mathrm{H} & 5.265034 & 0.107970 & -1.344005 & 1 & 9 & & & \\ 28 & \mathrm{Na} & -2.610869 & 3.276396 & -0.089251 & 1 & 2 & & & \end{array}$




\section{Geometry 15}

28

$\begin{array}{rrrrrrrrrr}1 & \mathrm{P} & 1.775368 & 0.073790 & -0.332526 & 1 & 5 & 4 & 3 & 2 \\ 2 & \mathrm{O} & 0.854958 & 1.281816 & -0.246299 & 1 & 28 & 1 & & \\ 3 & \mathrm{O} & 2.845412 & -0.057486 & 0.854873 & 1 & 9 & 1 & & \\ 4 & \mathrm{C} & 2.872794 & 0.111814 & -1.769177 & 1 & 17 & 16 & 15 & 1 \\ 5 & \mathrm{~S} & 0.681024 & -1.733148 & -0.337237 & 1 & 6 & 1 & & \\ 6 & \mathrm{C} & -1.047860 & -1.130560 & -0.630701 & 1 & 19 & 18 & 7 & 5 \\ 7 & \mathrm{C} & -1.808601 & -0.844938 & 0.683738 & 1 & 21 & 20 & 8 & 6 \\ 8 & \mathrm{~N} & -3.101599 & -0.189924 & 0.531652 & 1 & 11 & 10 & 7 & \\ 9 & \mathrm{C} & 2.496915 & -0.134768 & 2.258099 & 1 & 14 & 13 & 12 & 3 \\ 10 & \mathrm{C} & -4.098579 & -0.926337 & -0.241484 & 1 & 24 & 23 & 22 & 8 \\ 11 & \mathrm{C} & -3.056645 & 1.222862 & 0.241723 & 1 & 27 & 26 & 25 & 8 \\ 12 & \mathrm{H} & 2.035247 & 0.802149 & 2.581319 & 1 & 9 & & & \\ 13 & \mathrm{H} & 1.825455 & -0.979370 & 2.435850 & 1 & 9 & & & \\ 14 & \mathrm{H} & 3.438469 & -0.287762 & 2.784919 & 1 & 9 & & & \\ 15 & \mathrm{H} & 2.272042 & 0.156568 & -2.682078 & 1 & 4 & & & \\ 16 & \mathrm{H} & 3.515094 & 0.996132 & -1.704305 & 1 & 4 & & & \\ 17 & \mathrm{H} & 3.494781 & -0.787467 & -1.790591 & 1 & 4 & & & \\ 18 & \mathrm{H} & -1.519298 & -1.940439 & -1.194899 & 1 & 6 & & & \\ 19 & \mathrm{H} & -1.004703 & -0.256728 & -1.284775 & 1 & 6 & & & \\ 20 & \mathrm{H} & -1.187592 & -0.217908 & 1.334274 & 1 & 7 & & & \\ 21 & \mathrm{H} & -1.962656 & -1.792413 & 1.213875 & 1 & 7 & & & \\ 22 & \mathrm{H} & -3.909227 & -0.929362 & -1.329932 & 1 & 10 & & & \\ 23 & \mathrm{H} & -4.128244 & -1.963808 & 0.106500 & 1 & 10 & & & \\ 24 & \mathrm{H} & -5.087192 & -0.487500 & -0.071660 & 1 & 10 & & & \\ 25 & \mathrm{H} & -2.648374 & 1.479359 & -0.769063 & 1 & 11 & & & \\ 26 & \mathrm{H} & -4.066564 & 1.643778 & 0.278217 & 1 & 11 & & & \\ 27 & \mathrm{H} & -2.469406 & 1.730737 & 1.024906 & 1 & 11 & & & \\ 28 & \mathrm{Na} & -0.560421 & 2.836721 & -0.217474 & 1 & 2 & & & \\ & & & & & & & & \\ & \end{array}$




\section{Geometry 16}

28

\begin{tabular}{|c|c|c|c|c|c|c|c|c|c|}
\hline 1 & $\mathrm{P}$ & 1.328631 & -0.194963 & -1.083151 & 1 & 28 & 5 & 4 & 3 \\
\hline 2 & $\mathrm{O}$ & 0.556883 & 0.929894 & -1.738443 & 1 & 28 & 1 & & \\
\hline 3 & $\mathrm{O}$ & 2.119885 & 0.594331 & 0.146145 & 1 & 28 & 9 & 1 & \\
\hline 4 & $\mathrm{C}$ & 2.606782 & -0.984302 & -2.095463 & 1 & 17 & 16 & 15 & 1 \\
\hline 5 & S & 0.187050 & -1.773274 & -0.339029 & 1 & 6 & 1 & & \\
\hline 6 & $\mathrm{C}$ & -1.444449 & -0.948843 & 0.005810 & 1 & 19 & 18 & 7 & 5 \\
\hline 7 & $\mathrm{C}$ & -1.487704 & -0.200623 & 1.356478 & 1 & 21 & 20 & 8 & 6 \\
\hline 8 & $\mathrm{~N}$ & -2.779434 & 0.338006 & 1.735358 & 1 & 11 & 10 & 7 & \\
\hline 9 & $\mathrm{C}$ & 2.962224 & -0.078510 & 1.120765 & 1 & 14 & 13 & 12 & 3 \\
\hline 10 & $\mathrm{C}$ & -3.777724 & -0.654159 & 2.125075 & 1 & 24 & 23 & 22 & 8 \\
\hline 11 & $\mathrm{C}$ & -3.309294 & 1.374788 & 0.860453 & 1 & 27 & 26 & 25 & 8 \\
\hline 12 & $\mathrm{H}$ & 2.405105 & -0.887940 & 1.599817 & 1 & 9 & & & \\
\hline 13 & $\mathrm{H}$ & 3.864051 & -0.463246 & 0.636661 & 1 & 9 & & & \\
\hline 14 & $\mathrm{H}$ & 3.234479 & 0.674208 & 1.860906 & 1 & 9 & & & \\
\hline 15 & $\mathrm{H}$ & 2.134097 & -1.401571 & -2.989692 & 1 & 4 & & & \\
\hline 16 & $\mathrm{H}$ & 3.344556 & -0.232842 & -2.394811 & 1 & 4 & & & \\
\hline 17 & $\mathrm{H}$ & 3.105967 & -1.792025 & -1.550382 & 1 & 4 & & & \\
\hline 18 & $\mathrm{H}$ & -2.142949 & -1.791117 & -0.006882 & 1 & 6 & & & \\
\hline 19 & $\mathrm{H}$ & -1.674277 & -0.304405 & -0.845709 & 1 & 6 & & & \\
\hline 20 & $\mathrm{H}$ & -0.765597 & 0.629877 & 1.324876 & 1 & 7 & & & \\
\hline 21 & $\mathrm{H}$ & -1.153159 & -0.879287 & 2.150174 & 1 & 7 & & & \\
\hline 22 & $\mathrm{H}$ & -4.193814 & -1.229892 & 1.278263 & 1 & 10 & & & \\
\hline 23 & $\mathrm{H}$ & -3.338453 & -1.357026 & 2.840866 & 1 & 10 & & & \\
\hline 24 & $\mathrm{H}$ & -4.611294 & -0.146500 & 2.621362 & 1 & 10 & & & \\
\hline 25 & $\mathrm{H}$ & -3.673068 & 1.006017 & -0.116205 & 1 & 11 & & & \\
\hline 26 & $\mathrm{H}$ & -4.147680 & 1.872818 & 1.358701 & 1 & 11 & & & \\
\hline 27 & $\mathrm{H}$ & -2.535752 & 2.131571 & 0.674863 & 1 & 11 & & & \\
\hline 28 & $\mathrm{Na}$ & 1.088040 & 2.714385 & -0.583537 & 1 & 3 & 2 & 1 & \\
\hline
\end{tabular}




\section{Geometry 17}

28

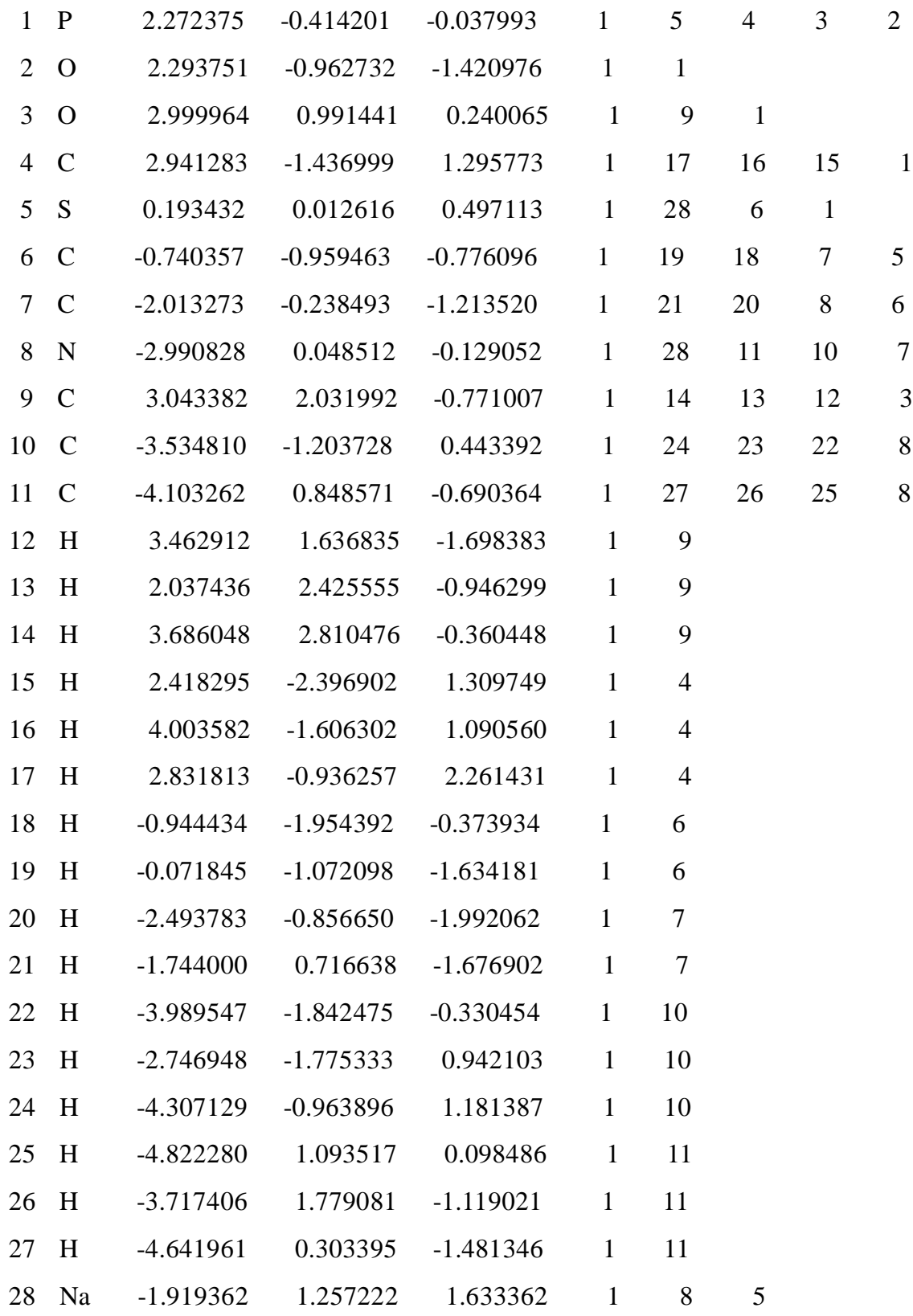




\section{Geometry 18}

28

$\begin{array}{rrrrrrrrrr}1 & \mathrm{P} & -1.611254 & -0.679734 & 0.089637 & 1 & 5 & 4 & 3 & 2 \\ 2 & \mathrm{O} & -0.847632 & -1.548884 & 1.023775 & 1 & 1 & & & \\ 3 & \mathrm{O} & -2.063483 & 0.790126 & 0.758271 & 1 & 28 & 9 & 1 & \\ 4 & \mathrm{C} & -3.131572 & -1.369780 & -0.619684 & 1 & 17 & 16 & 15 & 1 \\ 5 & \mathrm{~S} & -0.463770 & 0.071716 & -1.552997 & 1 & 28 & 6 & 1 & \\ 6 & \mathrm{C} & 1.238014 & -0.490272 & -1.041453 & 1 & 19 & 18 & 7 & 5 \\ 7 & \mathrm{C} & 1.911897 & 0.494000 & -0.056880 & 1 & 21 & 20 & 8 & 6 \\ 8 & \mathrm{~N} & 3.267417 & 0.195544 & 0.334005 & 1 & 11 & 10 & 7 & \\ 9 & \mathrm{C} & -2.871584 & 0.751386 & 1.980345 & 1 & 14 & 13 & 12 & 3 \\ 10 & \mathrm{C} & 4.266234 & 0.307074 & -0.725263 & 1 & 24 & 23 & 22 & 8 \\ 11 & \mathrm{C} & 3.431422 & -0.992156 & 1.170623 & 1 & 27 & 26 & 25 & 8 \\ 12 & \mathrm{H} & -3.886233 & 0.424407 & 1.740442 & 1 & 9 & & & \\ 13 & \mathrm{H} & -2.407539 & 0.085583 & 2.711066 & 1 & 9 & & & \\ 14 & \mathrm{H} & -2.900291 & 1.771403 & 2.365574 & 1 & 9 & & & \\ 15 & \mathrm{H} & -2.871290 & -2.274276 & -1.177562 & 1 & 4 & & & \\ 16 & \mathrm{H} & -3.815540 & -1.647737 & 0.189087 & 1 & 4 & & & \\ 17 & \mathrm{H} & -3.618985 & -0.654861 & -1.289489 & 1 & 4 & & & \\ 18 & \mathrm{H} & 1.781285 & -0.563698 & -1.987539 & 1 & 6 & & & \\ 19 & \mathrm{H} & 1.133911 & -1.488889 & -0.611676 & 1 & 6 & & & \\ 20 & \mathrm{H} & 1.299929 & 0.530334 & 0.858184 & 1 & 7 & & & \\ 21 & \mathrm{H} & 1.911870 & 1.496950 & -0.520909 & 1 & 7 & & & \\ 22 & \mathrm{H} & 4.234135 & -0.517251 & -1.460100 & 1 & 10 & & & \\ 23 & \mathrm{H} & 4.136718 & 1.255124 & -1.259947 & 1 & 10 & & & \\ 24 & \mathrm{H} & 5.263721 & 0.310290 & -0.274586 & 1 & 10 & & & \\ 25 & \mathrm{H} & 3.333367 & -1.942459 & 0.617208 & 1 & 11 & & & \\ 26 & \mathrm{H} & 4.427218 & -0.970854 & 1.624685 & 1 & 11 & & & \\ 27 & \mathrm{H} & 2.689172 & -0.984575 & 1.975508 & 1 & 11 & & & \\ 28 & \mathrm{Na} & -0.706525 & 2.428982 & -0.076098 & 1 & 5 & 3 & & \end{array}$




\section{Geometry 19}

28

$\begin{array}{rrrrrrrrrr}1 & \mathrm{P} & -1.704323 & 0.050822 & 0.458995 & 1 & 5 & 4 & 3 & 2 \\ 2 & \mathrm{O} & -0.835164 & 1.031548 & 1.196018 & 1 & 28 & 1 & & \\ 3 & \mathrm{O} & -2.787646 & 0.817221 & -0.450923 & 1 & 9 & 1 & & \\ 4 & \mathrm{C} & -2.582707 & -1.103997 & 1.550515 & 1 & 17 & 16 & 15 & 1 \\ 5 & \mathrm{~S} & -0.597385 & -1.007105 & -1.012726 & 1 & 6 & 1 & & \\ 6 & \mathrm{C} & 0.706878 & -1.866429 & 0.003824 & 1 & 19 & 18 & 7 & 5 \\ 7 & \mathrm{C} & 2.132892 & -1.542298 & -0.456635 & 1 & 21 & 20 & 8 & 6 \\ 8 & \mathrm{~N} & 2.659727 & -0.227938 & -0.041731 & 1 & 28 & 11 & 10 & 7 \\ 9 & \mathrm{C} & -3.935244 & 0.208293 & -1.093739 & 1 & 14 & 13 & 12 & 3 \\ 10 & \mathrm{C} & 3.890154 & 0.060719 & -0.802370 & 1 & 24 & 23 & 22 & 8 \\ 11 & \mathrm{C} & 2.958898 & -0.211023 & 1.401915 & 1 & 27 & 26 & 25 & 8 \\ 12 & \mathrm{H} & -3.625539 & -0.620924 & -1.736517 & 1 & 9 & & & \\ 13 & \mathrm{H} & -4.651588 & -0.133693 & -0.341828 & 1 & 9 & & & \\ 14 & \mathrm{H} & -4.385337 & 0.996514 & -1.697003 & 1 & 9 & & & \\ 15 & \mathrm{H} & -1.858342 & -1.657909 & 2.155011 & 1 & 4 & & & \\ 16 & \mathrm{H} & -3.218203 & -0.519133 & 2.224955 & 1 & 4 & & & \\ 17 & \mathrm{H} & -3.191968 & -1.814447 & 0.985435 & 1 & 4 & & & \\ 18 & \mathrm{H} & 0.530980 & -2.937532 & -0.124628 & 1 & 6 & & & \\ 19 & \mathrm{H} & 0.558422 & -1.631577 & 1.060332 & 1 & 6 & & & \\ 20 & \mathrm{H} & 2.174763 & -1.596734 & -1.549760 & 1 & 7 & & & \\ 21 & \mathrm{H} & 2.788662 & -2.348358 & -0.077114 & 1 & 7 & & & \\ 22 & \mathrm{H} & 4.674734 & -0.697139 & -0.636957 & 1 & 10 & & & \\ 23 & \mathrm{H} & 3.668282 & 0.096265 & -1.873907 & 1 & 10 & & & \\ 24 & \mathrm{H} & 4.298159 & 1.030991 & -0.497120 & 1 & 10 & & & \\ 25 & \mathrm{H} & 3.681176 & -0.995347 & 1.686555 & 1 & 11 & & & \\ 26 & \mathrm{H} & 3.395500 & 0.755790 & 1.677581 & 1 & 11 & & & \\ 27 & \mathrm{H} & 2.047310 & -0.347411 & 1.990457 & 1 & 11 & & & \\ 28 & \mathrm{~K} & 1.030271 & 2.173990 & -0.169290 & 1 & 8 & 2 & & \end{array}$




\section{Geometry 20}

28

$\begin{array}{rrrrrrrrrr}1 & \mathrm{P} & 1.221228 & 0.419935 & 0.618568 & 1 & 5 & 4 & 3 & 2 \\ 2 & \mathrm{O} & 2.619306 & -0.013293 & 0.221723 & 1 & 28 & 1 & & \\ 3 & \mathrm{O} & 0.831203 & 1.925076 & 0.185286 & 1 & 9 & 1 & & \\ 4 & \mathrm{C} & 0.947115 & 0.515264 & 2.405651 & 1 & 17 & 16 & 15 & 1 \\ 5 & \mathrm{~S} & -0.112285 & -0.917539 & -0.291664 & 1 & 6 & 1 & & \\ 6 & \mathrm{C} & -1.785495 & -0.240051 & 0.156173 & 1 & 19 & 18 & 7 & 5 \\ 7 & \mathrm{C} & -2.847540 & -0.939034 & -0.734500 & 1 & 21 & 20 & 8 & 6 \\ 8 & \mathrm{~N} & -4.219815 & -0.542086 & -0.487231 & 1 & 11 & 10 & 7 & \\ 9 & \mathrm{C} & 1.013774 & 2.411729 & -1.163824 & 1 & 14 & 13 & 12 & 3 \\ 10 & \mathrm{C} & -4.546034 & 0.833109 & -0.839701 & 1 & 24 & 23 & 22 & 8 \\ 11 & \mathrm{C} & -4.772378 & -0.958072 & 0.795234 & 1 & 27 & 26 & 25 & 8 \\ 12 & \mathrm{H} & 2.080267 & 2.486475 & -1.392859 & 1 & 9 & & & \\ 13 & \mathrm{H} & 0.515562 & 1.752112 & -1.881274 & 1 & 9 & & & \\ 14 & \mathrm{H} & 0.558549 & 3.401946 & -1.187085 & 1 & 9 & & & \\ 15 & \mathrm{H} & 1.149335 & -0.461984 & 2.852885 & 1 & 4 & & & \\ 16 & \mathrm{H} & 1.630999 & 1.260663 & 2.824569 & 1 & 4 & & & \\ 17 & \mathrm{H} & -0.081159 & 0.813408 & 2.627720 & 1 & 4 & & & \\ 18 & \mathrm{H} & -1.781682 & 0.839848 & -0.010508 & 1 & 6 & & & \\ 19 & \mathrm{H} & -1.971042 & -0.442148 & 1.214323 & 1 & 6 & & & \\ 20 & \mathrm{H} & -2.774720 & -2.023995 & -0.588280 & 1 & 7 & & & \\ 21 & \mathrm{H} & -2.612334 & -0.736923 & -1.787046 & 1 & 7 & & & \\ 22 & \mathrm{H} & -4.141711 & 1.587293 & -0.137272 & 1 & 10 & & & \\ 23 & \mathrm{H} & -4.168166 & 1.056799 & -1.843738 & 1 & 10 & & & \\ 24 & \mathrm{H} & -5.634726 & 0.951280 & -0.856988 & 1 & 10 & & & \\ 25 & \mathrm{H} & -4.393057 & -0.379892 & 1.659843 & 1 & 11 & & & \\ 26 & \mathrm{H} & -5.860958 & -0.839062 & 0.773213 & 1 & 11 & & & \\ 27 & \mathrm{H} & -4.553773 & -2.017999 & 0.965261 & 1 & 11 & & & \\ 28 & \mathrm{~K} & 4.704987 & -1.058082 & -0.600227 & 1 & 2 & & & \end{array}$




\section{Geometry 21}

28

$\begin{array}{rrrrrrrrrr}1 & \mathrm{P} & -1.746771 & -0.488314 & 0.015605 & 1 & 11 & 4 & 3 & 2 \\ 2 & \mathrm{O} & -1.280681 & -1.440431 & 1.066160 & 1 & 1 & & & \\ 3 & \mathrm{O} & -1.798121 & 1.093788 & 0.508394 & 1 & 28 & 5 & 1 & \\ 4 & \mathrm{C} & -3.381528 & -0.834532 & -0.696067 & 1 & 15 & 14 & 13 & 1 \\ 5 & \mathrm{C} & -2.764894 & 1.465535 & 1.534051 & 1 & 24 & 17 & 16 & 3 \\ 6 & \mathrm{C} & 0.853260 & -1.597525 & -1.209543 & 1 & 19 & 18 & 11 & 7 \\ 7 & \mathrm{C} & 2.273593 & -1.085373 & -0.961820 & 1 & 21 & 20 & 8 & 6 \\ 8 & \mathrm{~N} & 2.465415 & -0.244117 & 0.234457 & 1 & 28 & 10 & 9 & 7 \\ 9 & \mathrm{C} & 3.850407 & 0.262802 & 0.243776 & 1 & 27 & 22 & 12 & 8 \\ 10 & \mathrm{C} & 2.210694 & -1.003644 & 1.476514 & 1 & 26 & 25 & 23 & 8 \\ 11 & \mathrm{~S} & -0.378138 & -0.255320 & -1.600849 & 1 & 6 & 1 & & \\ 12 & \mathrm{H} & 4.047661 & 0.843455 & -0.664471 & 1 & 9 & & & \\ 13 & \mathrm{H} & -3.655847 & -0.080134 & -1.439542 & 1 & 4 & & & \\ 14 & \mathrm{H} & -3.347687 & -1.818121 & -1.173802 & 1 & 4 & & & \\ 15 & \mathrm{H} & -4.130567 & -0.865092 & 0.101926 & 1 & 4 & & & \\ 16 & \mathrm{H} & -3.778493 & 1.432868 & 1.125667 & 1 & 5 & & & \\ 17 & \mathrm{H} & -2.675630 & 0.803896 & 2.399823 & 1 & 5 & & & \\ 18 & \mathrm{H} & 0.457381 & -2.169238 & -0.367480 & 1 & 6 & & & \\ 19 & \mathrm{H} & 0.865641 & -2.248132 & -2.087692 & 1 & 6 & & & \\ 20 & \mathrm{H} & 2.919962 & -1.982109 & -0.906519 & 1 & 7 & & & \\ 21 & \mathrm{H} & 2.609856 & -0.512211 & -1.833878 & 1 & 7 & & & \\ 22 & \mathrm{H} & 4.004479 & 0.909243 & 1.115236 & 1 & 9 & & & \\ 23 & \mathrm{H} & 2.850099 & -1.900341 & 1.545893 & 1 & 10 & & & \\ 24 & \mathrm{H} & -2.529530 & 2.491504 & 1.821194 & 1 & 5 & & & \\ 25 & \mathrm{H} & 2.434180 & -0.368843 & 2.341127 & 1 & 10 & & & \\ 26 & \mathrm{H} & 1.163683 & -1.309213 & 1.539584 & 1 & 10 & & & \\ 27 & \mathrm{H} & 4.594803 & -0.549555 & 0.296330 & 1 & 9 & & & \\ 28 & \mathrm{~K} & 0.818163 & 2.103699 & 0.263527 & 1 & 8 & 3 & & \\ & & & & & & & & & \\ & \end{array}$




\section{Geometry 22}

28

$\begin{array}{rrrrrrrrrr}1 & \mathrm{P} & 1.532638 & -0.547689 & -0.431299 & 1 & 5 & 4 & 3 & 2 \\ 2 & \mathrm{O} & 1.207429 & 0.656613 & -1.279857 & 1 & 28 & 1 & & \\ 3 & \mathrm{O} & 1.823851 & 0.079079 & 1.066757 & 1 & 9 & 1 & & \\ 4 & \mathrm{C} & 3.025354 & -1.466882 & -0.899055 & 1 & 17 & 16 & 15 & 1 \\ 5 & \mathrm{~S} & 0.040510 & -2.013436 & -0.334393 & 1 & 6 & 1 & & \\ 6 & \mathrm{C} & -1.496574 & -1.016624 & -0.637279 & 1 & 19 & 18 & 7 & 5 \\ 7 & \mathrm{C} & -2.036073 & -0.319104 & 0.630714 & 1 & 21 & 20 & 8 & 6 \\ 8 & \mathrm{~N} & -3.311686 & 0.359120 & 0.486286 & 1 & 11 & 10 & 7 & \\ 9 & \mathrm{C} & 2.126826 & -0.735333 & 2.227950 & 1 & 14 & 13 & 12 & 3 \\ 10 & \mathrm{C} & -4.465802 & -0.519013 & 0.314964 & 1 & 24 & 23 & 22 & 8 \\ 11 & \mathrm{C} & -3.316341 & 1.494921 & -0.424066 & 1 & 27 & 26 & 25 & 8 \\ 12 & \mathrm{H} & 1.347473 & -1.487758 & 2.378051 & 1 & 9 & & & \\ 13 & \mathrm{H} & 3.104312 & -1.213108 & 2.113515 & 1 & 9 & & & \\ 14 & \mathrm{H} & 2.150197 & -0.054414 & 3.079458 & 1 & 9 & & & \\ 15 & \mathrm{H} & 2.927225 & -1.784845 & -1.941177 & 1 & 4 & & & \\ 16 & \mathrm{H} & 3.899528 & -0.815015 & -0.800776 & 1 & 4 & & & \\ 17 & \mathrm{H} & 3.161584 & -2.354656 & -0.272692 & 1 & 4 & & & \\ 18 & \mathrm{H} & -2.201072 & -1.765398 & -1.012121 & 1 & 6 & & & \\ 19 & \mathrm{H} & -1.283369 & -0.315883 & -1.447833 & 1 & 6 & & & \\ 20 & \mathrm{H} & -1.293702 & 0.417562 & 0.971170 & 1 & 7 & & & \\ 21 & \mathrm{H} & -2.133236 & -1.062659 & 1.430797 & 1 & 7 & & & \\ 22 & \mathrm{H} & -4.528181 & -0.990272 & -0.683035 & 1 & 10 & & & \\ 23 & \mathrm{H} & -4.440932 & -1.310519 & 1.071377 & 1 & 10 & & & \\ 24 & \mathrm{H} & -5.382607 & 0.060782 & 0.465165 & 1 & 10 & & & \\ 25 & \mathrm{H} & -3.245168 & 1.224071 & -1.493911 & 1 & 11 & & & \\ 26 & \mathrm{H} & -4.243079 & 2.062479 & -0.287956 & 1 & 11 & & & \\ 27 & \mathrm{H} & -2.478376 & 2.162468 & -0.182346 & 1 & 11 & & & \\ 28 & \mathrm{~K} & 1.416258 & 2.875280 & -0.028762 & 1 & 2 & & & \end{array}$




\section{Geometry 23}

28

$\begin{array}{rrrrrrrrrr}1 & \mathrm{P} & -1.683666 & -0.373207 & 0.424729 & 1 & 5 & 4 & 3 & 2 \\ 2 & \mathrm{O} & -1.074297 & 0.995313 & 0.176601 & 1 & 28 & 1 & & \\ 3 & \mathrm{O} & -3.031378 & -0.690122 & -0.392782 & 1 & 9 & 1 & & \\ 4 & \mathrm{C} & -2.261236 & -0.591570 & 2.128509 & 1 & 17 & 16 & 15 & 1 \\ 5 & \mathrm{~S} & -0.416217 & -1.967758 & -0.115141 & 1 & 6 & 1 & & \\ 6 & \mathrm{C} & 1.259163 & -1.350289 & 0.393538 & 1 & 19 & 18 & 7 & 5 \\ 7 & \mathrm{C} & 1.935393 & -0.484350 & -0.691668 & 1 & 21 & 20 & 8 & 6 \\ 8 & \mathrm{~N} & 3.295684 & -0.049730 & -0.410121 & 1 & 11 & 10 & 7 & \\ 9 & \mathrm{C} & -3.139614 & -0.593984 & -1.832966 & 1 & 14 & 13 & 12 & 3 \\ 10 & \mathrm{C} & 4.304810 & -1.105218 & -0.454224 & 1 & 24 & 23 & 22 & 8 \\ 11 & \mathrm{C} & 3.442356 & 0.822560 & 0.745083 & 1 & 27 & 26 & 25 & 8 \\ 12 & \mathrm{H} & -3.032841 & 0.448165 & -2.146711 & 1 & 9 & & & \\ 13 & \mathrm{H} & -2.384741 & -1.218534 & -2.318185 & 1 & 9 & & & \\ 14 & \mathrm{H} & -4.138039 & -0.956770 & -2.076548 & 1 & 9 & & & \\ 15 & \mathrm{H} & -1.415446 & -0.501069 & 2.816688 & 1 & 4 & & & \\ 16 & \mathrm{H} & -2.999051 & 0.184794 & 2.356311 & 1 & 4 & & & \\ 17 & \mathrm{H} & -2.719301 & -1.576893 & 2.247261 & 1 & 4 & & & \\ 18 & \mathrm{H} & 1.822563 & -2.269638 & 0.579184 & 1 & 6 & & & \\ 19 & \mathrm{H} & 1.172705 & -0.823071 & 1.347588 & 1 & 6 & & & \\ 20 & \mathrm{H} & 1.312826 & 0.404579 & -0.854255 & 1 & 7 & & & \\ 21 & \mathrm{H} & 1.944390 & -1.042291 & -1.635197 & 1 & 7 & & & \\ 22 & \mathrm{H} & 4.264038 & -1.801065 & 0.403680 & 1 & 10 & & & \\ 23 & \mathrm{H} & 4.189753 & -1.684334 & -1.376269 & 1 & 10 & & & \\ 24 & \mathrm{H} & 5.300799 & -0.649895 & -0.464822 & 1 & 10 & & & \\ 25 & \mathrm{H} & 3.322107 & 0.315555 & 1.721196 & 1 & 11 & & & \\ 26 & \mathrm{H} & 4.437898 & 1.279513 & 0.732338 & 1 & 11 & & & \\ 27 & \mathrm{H} & 2.701747 & 1.633350 & 0.692025 & 1 & 11 & & & \\ 28 & \mathrm{~K} & -0.280766 & 3.319133 & -0.193806 & 1 & 2 & & & \end{array}$




\section{Geometry 24}

28

$\begin{array}{rrrrrrrrrr}1 & \mathrm{P} & -1.610291 & -0.484345 & 0.063574 & 1 & 5 & 4 & 3 & 2 \\ 2 & \mathrm{O} & -1.144419 & 0.704206 & 0.881850 & 1 & 28 & 1 & & \\ 3 & \mathrm{O} & -2.965286 & -0.242424 & -0.772245 & 1 & 9 & 1 & & \\ 4 & \mathrm{C} & -1.868896 & -1.946520 & 1.117660 & 1 & 17 & 16 & 15 & 1 \\ 5 & \mathrm{~S} & -0.407867 & -0.980196 & -1.588011 & 1 & 6 & 1 & & \\ 6 & \mathrm{C} & 1.272449 & -1.183852 & -0.827291 & 1 & 19 & 18 & 7 & 5 \\ 7 & \mathrm{C} & 1.952656 & 0.147776 & -0.439507 & 1 & 21 & 20 & 8 & 6 \\ 8 & \mathrm{~N} & 3.355857 & 0.088220 & -0.063961 & 1 & 11 & 10 & 7 & \\ 9 & \mathrm{C} & -4.274008 & -0.139184 & -0.165858 & 1 & 14 & 13 & 12 & 3 \\ 10 & \mathrm{C} & 4.269242 & -0.201427 & -1.168069 & 1 & 24 & 23 & 22 & 8 \\ 11 & \mathrm{C} & 3.642095 & -0.708366 & 1.125520 & 1 & 27 & 26 & 25 & 8 \\ 12 & \mathrm{H} & -4.556775 & -1.087841 & 0.300473 & 1 & 9 & & & \\ 13 & \mathrm{H} & -4.295133 & 0.669289 & 0.571190 & 1 & 9 & & & \\ 14 & \mathrm{H} & -4.959248 & 0.085871 & -0.982938 & 1 & 9 & & & \\ 15 & \mathrm{H} & -0.911048 & -2.276471 & 1.531730 & 1 & 4 & & & \\ 16 & \mathrm{H} & -2.526571 & -1.682692 & 1.952535 & 1 & 4 & & & \\ 17 & \mathrm{H} & -2.306561 & -2.764707 & 0.538986 & 1 & 4 & & & \\ 18 & \mathrm{H} & 1.829424 & -1.690993 & -1.621308 & 1 & 6 & & & \\ 19 & \mathrm{H} & 1.212707 & -1.877318 & 0.016302 & 1 & 6 & & & \\ 20 & \mathrm{H} & 1.395151 & 0.567940 & 0.408771 & 1 & 7 & & & \\ 21 & \mathrm{H} & 1.854271 & 0.843748 & -1.282628 & 1 & 7 & & & \\ 22 & \mathrm{H} & 4.220650 & -1.245862 & -1.526066 & 1 & 10 & & & \\ 23 & \mathrm{H} & 4.053168 & 0.463667 & -2.011043 & 1 & 10 & & & \\ 24 & \mathrm{H} & 5.296917 & -0.010911 & -0.842017 & 1 & 10 & & & \\ 25 & \mathrm{H} & 3.556510 & -1.798822 & 0.967344 & 1 & 11 & & & \\ 26 & \mathrm{H} & 4.664961 & -0.502743 & 1.458207 & 1 & 11 & & & \\ 27 & \mathrm{H} & 2.960031 & -0.425286 & 1.936744 & 1 & 11 & & & \\ 28 & \mathrm{~K} & -0.072779 & 2.924162 & 1.302865 & 1 & 2 & & & \\ & & & & & & & & \\ & \end{array}$




\section{Geometry 25}

28

$\begin{array}{rrrrrrrrrr}1 & \mathrm{P} & -2.309411 & -0.098212 & -0.842457 & 1 & 5 & 4 & 3 & 2 \\ 2 & \mathrm{O} & -2.419636 & -1.559353 & -1.108781 & 1 & 1 & & & \\ 3 & \mathrm{O} & -3.163222 & 0.510581 & 0.383907 & 1 & 9 & 1 & & \\ 4 & \mathrm{C} & -2.762838 & 1.036509 & -2.177913 & 1 & 17 & 16 & 15 & 1 \\ 5 & \mathrm{~S} & -0.264601 & 0.375691 & -0.282812 & 1 & 6 & 1 & & \\ 6 & \mathrm{C} & 0.634157 & -1.146400 & -0.847619 & 1 & 19 & 18 & 7 & 5 \\ 7 & \mathrm{C} & 1.713266 & -1.568087 & 0.145793 & 1 & 21 & 20 & 8 & 6 \\ 8 & \mathrm{~N} & 2.787046 & -0.575386 & 0.382339 & 1 & 28 & 11 & 10 & 7 \\ 9 & \mathrm{C} & -3.415128 & -0.278642 & 1.572845 & 1 & 14 & 13 & 12 & 3 \\ 10 & \mathrm{C} & 3.599359 & -0.364533 & -0.830763 & 1 & 24 & 23 & 22 & 8 \\ 11 & \mathrm{C} & 3.654096 & -1.059135 & 1.474980 & 1 & 27 & 26 & 25 & 8 \\ 12 & \mathrm{H} & -3.861079 & -1.237882 & 1.301448 & 1 & 9 & & & \\ 13 & \mathrm{H} & -2.482529 & -0.439400 & 2.123770 & 1 & 9 & & & \\ 14 & \mathrm{H} & -4.109551 & 0.308636 & 2.173849 & 1 & 9 & & & \\ 15 & \mathrm{H} & -2.154375 & 0.816030 & -3.059170 & 1 & 4 & & & \\ 16 & \mathrm{H} & -3.818604 & 0.871736 & -2.417478 & 1 & 4 & & & \\ 17 & \mathrm{H} & -2.617031 & 2.076599 & -1.874304 & 1 & 4 & & & \\ 18 & \mathrm{H} & 1.035144 & -0.960280 & -1.846391 & 1 & 6 & & & \\ 19 & \mathrm{H} & -0.118207 & -1.936161 & -0.927070 & 1 & 6 & & & \\ 20 & \mathrm{H} & 2.147924 & -2.515954 & -0.222809 & 1 & 7 & & & \\ 21 & \mathrm{H} & 1.241987 & -1.787477 & 1.110089 & 1 & 7 & & & \\ 22 & \mathrm{H} & 4.054288 & -1.300629 & -1.195451 & 1 & 10 & & & \\ 23 & \mathrm{H} & 2.991213 & 0.058712 & -1.634999 & 1 & 10 & & & \\ 24 & \mathrm{H} & 4.411540 & 0.338652 & -0.613139 & 1 & 10 & & & \\ 25 & \mathrm{H} & 4.444544 & -0.326776 & 1.675319 & 1 & 11 & & & \\ 26 & \mathrm{H} & 3.062134 & -1.201460 & 2.386429 & 1 & 11 & & & \\ 27 & \mathrm{H} & 4.140594 & -2.018234 & 1.232060 & 1 & 11 & & & \\ 28 & \mathrm{~K} & 1.848572 & 1.969244 & 1.370968 & 1 & 8 & & & \end{array}$




\section{Geometry 26}

28

$\begin{array}{rrrrrrrrrr}1 & \mathrm{P} & 1.481424 & -0.292545 & 0.270204 & 1 & 5 & 4 & 3 & 2 \\ 2 & \mathrm{O} & 0.365218 & -1.391937 & 0.223036 & 1 & 28 & 1 & & \\ 3 & \mathrm{O} & 2.541603 & -0.584656 & -0.825196 & 1 & 9 & 1 & & \\ 4 & \mathrm{C} & 2.156297 & -0.014266 & 1.912099 & 1 & 17 & 16 & 15 & 1 \\ 5 & \mathrm{~S} & 0.229268 & 1.288907 & -0.574519 & 1 & 28 & 6 & 1 & \\ 6 & \mathrm{C} & -1.132635 & 1.639636 & 0.700787 & 1 & 19 & 18 & 7 & 5 \\ 7 & \mathrm{C} & -2.455581 & 1.116938 & 0.123511 & 1 & 21 & 20 & 8 & 6 \\ 8 & \mathrm{~N} & -2.390040 & -0.370666 & -0.184681 & 1 & 28 & 11 & 10 & 7 \\ 9 & \mathrm{C} & 3.944862 & -0.124137 & -1.017371 & 1 & 14 & 13 & 12 & 3 \\ 10 & \mathrm{C} & -3.267991 & -0.701159 & -1.372346 & 1 & 24 & 23 & 22 & 8 \\ 11 & \mathrm{C} & -2.850584 & -1.183160 & 1.007408 & 1 & 27 & 26 & 25 & 8 \\ 12 & \mathrm{H} & 3.933990 & 0.941920 & -1.245127 & 1 & 9 & & & \\ 13 & \mathrm{H} & 4.519476 & -0.354959 & -0.120247 & 1 & 9 & & & \\ 14 & \mathrm{H} & 4.294739 & -0.705786 & -1.867726 & 1 & 9 & & & \\ 15 & \mathrm{H} & 1.356605 & 0.190456 & 2.630568 & 1 & 4 & & & \\ 16 & \mathrm{H} & 2.671860 & -0.936262 & 2.215523 & 1 & 4 & & & \\ 17 & \mathrm{H} & 2.866085 & 0.820089 & 1.901301 & 1 & 4 & & & \\ 18 & \mathrm{H} & -1.156671 & 2.726156 & 0.818591 & 1 & 6 & & & \\ 19 & \mathrm{H} & -0.871602 & 1.196184 & 1.665580 & 1 & 6 & & & \\ 20 & \mathrm{H} & -2.688562 & 1.643864 & -0.805654 & 1 & 7 & & & \\ 21 & \mathrm{H} & -3.268648 & 1.310679 & 0.832277 & 1 & 7 & & & \\ 22 & \mathrm{H} & -4.297640 & -0.396884 & -1.161989 & 1 & 10 & & & \\ 23 & \mathrm{H} & -2.908431 & -0.173482 & -2.259520 & 1 & 10 & & & \\ 24 & \mathrm{H} & -3.242963 & -1.779373 & -1.546720 & 1 & 10 & & & \\ 25 & \mathrm{H} & -3.911465 & -0.988401 & 1.189022 & 1 & 11 & & & \\ 26 & \mathrm{H} & -2.707951 & -2.245596 & 0.798639 & 1 & 11 & & & \\ 27 & \mathrm{H} & -2.273876 & -0.910705 & 1.894138 & 1 & 11 & & & \\ 28 & \mathrm{Be} & -0.773771 & -0.641984 & -0.453475 & 1 & 8 & 5 & 2 & \end{array}$




\section{Geometry 27}

28

$\begin{array}{rrrrrrrrrr}1 & \mathrm{P} & -1.740757 & -0.177877 & 0.237334 & 1 & 11 & 4 & 3 & 2 \\ 2 & \mathrm{O} & -1.659714 & -0.143715 & 1.707785 & 1 & 1 & & & \\ 3 & \mathrm{O} & -0.809803 & 1.165343 & -0.468554 & 1 & 28 & 5 & 1 & \\ 4 & \mathrm{C} & -3.299844 & -0.243799 & -0.660524 & 1 & 15 & 14 & 13 & 1 \\ 5 & \mathrm{C} & -1.137496 & 2.595116 & -0.142168 & 1 & 24 & 17 & 16 & 3 \\ 6 & \mathrm{C} & 0.954490 & -1.837243 & 0.645799 & 1 & 19 & 18 & 11 & 7 \\ 7 & \mathrm{C} & 2.265701 & -1.156367 & 0.237542 & 1 & 21 & 20 & 8 & 6 \\ 8 & \mathrm{~N} & 2.066407 & 0.319745 & -0.090722 & 1 & 28 & 10 & 9 & 7 \\ 9 & \mathrm{C} & 3.151239 & 0.782257 & -1.040453 & 1 & 27 & 22 & 12 & 8 \\ 10 & \mathrm{C} & 2.137557 & 1.145011 & 1.177224 & 1 & 26 & 25 & 23 & 8 \\ 11 & \mathrm{~S} & -0.291830 & -1.521215 & -0.739366 & 1 & 28 & 6 & 1 & \\ 12 & \mathrm{H} & 3.081916 & 0.231840 & -1.982425 & 1 & 9 & & & \\ 13 & \mathrm{H} & -3.142894 & -0.204382 & -1.743182 & 1 & 4 & & & \\ 14 & \mathrm{H} & -3.798708 & -1.181452 & -0.383868 & 1 & 4 & & & \\ 15 & \mathrm{H} & -3.925484 & 0.594588 & -0.329303 & 1 & 4 & & & \\ 16 & \mathrm{H} & -2.084137 & 2.827143 & -0.628617 & 1 & 5 & & & \\ 17 & \mathrm{H} & -1.191882 & 2.690252 & 0.942470 & 1 & 5 & & & \\ 18 & \mathrm{H} & 0.520448 & -1.476959 & 1.583734 & 1 & 6 & & & \\ 19 & \mathrm{H} & 1.073726 & -2.921993 & 0.707363 & 1 & 6 & & & \\ 20 & \mathrm{H} & 3.001416 & -1.252790 & 1.044034 & 1 & 7 & & & \\ 21 & \mathrm{H} & 2.677765 & -1.638924 & -0.652898 & 1 & 7 & & & \\ 22 & \mathrm{H} & 3.036496 & 1.852720 & -1.226506 & 1 & 9 & & & \\ 23 & \mathrm{H} & 3.131202 & 1.038228 & 1.622314 & 1 & 10 & & & \\ 24 & \mathrm{H} & -0.333169 & 3.194417 & -0.566115 & 1 & 5 & & & \\ 25 & \mathrm{H} & 1.972946 & 2.197348 & 0.935129 & 1 & 10 & & & \\ 26 & \mathrm{H} & 1.383189 & 0.808936 & 1.892859 & 1 & 10 & & & \\ 27 & \mathrm{H} & 4.132297 & 0.603197 & -0.590492 & 1 & 9 & & & \\ 28 & \mathrm{Be} & 0.526728 & 0.381088 & -0.734491 & 1 & 11 & 8 & 3 & \end{array}$




\section{Geometry 28}

28

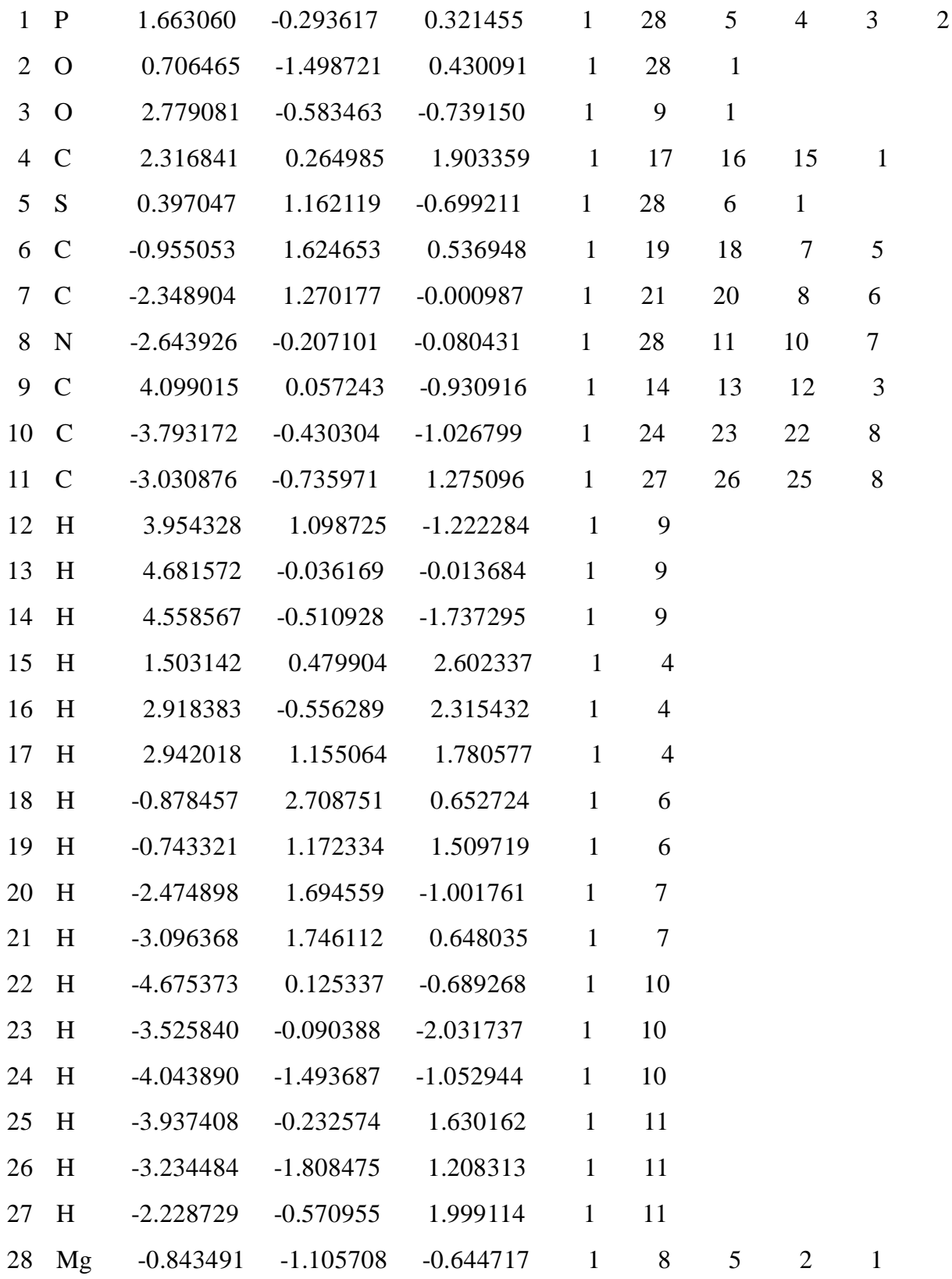




\section{Geometry 29}

28

$\begin{array}{rlrrrrrrrr}1 & \mathrm{P} & -1.732140 & -0.287622 & 0.330459 & 1 & 11 & 4 & 3 & 2 \\ 2 & \mathrm{O} & -1.359737 & -0.250008 & 1.762283 & 1 & 1 & & & \\ 3 & \mathrm{O} & -1.314420 & 1.196427 & -0.458947 & 1 & 28 & 5 & 1 & \\ 4 & \mathrm{C} & -3.406755 & -0.699762 & -0.193371 & 1 & 15 & 14 & 13 & 1 \\ 5 & \mathrm{C} & -2.094007 & 2.426983 & -0.113168 & 1 & 24 & 17 & 16 & 3 \\ 6 & \mathrm{C} & 1.013732 & -1.830676 & 0.414444 & 1 & 19 & 18 & 11 & 7 \\ 7 & \mathrm{C} & 2.344147 & -1.143570 & 0.083832 & 1 & 21 & 20 & 8 & 6 \\ 8 & \mathrm{~N} & 2.305558 & 0.364323 & 0.050134 & 1 & 28 & 10 & 9 & 7 \\ 9 & \mathrm{C} & 3.591941 & 0.867431 & -0.548392 & 1 & 27 & 22 & 12 & 8 \\ 10 & \mathrm{C} & 2.171403 & 0.917997 & 1.445277 & 1 & 26 & 25 & 23 & 8 \\ 11 & \mathrm{~S} & -0.317388 & -1.464022 & -0.867942 & 1 & 28 & 6 & 1 & \\ 12 & \mathrm{H} & 3.705684 & 0.493313 & -1.569932 & 1 & 9 & & & \\ 13 & \mathrm{H} & -3.516252 & -0.632140 & -1.280322 & 1 & 4 & & & \\ 14 & \mathrm{H} & -3.616980 & -1.722982 & 0.139533 & 1 & 4 & & & \\ 15 & \mathrm{H} & -4.110551 & -0.024204 & 0.306934 & 1 & 4 & & & \\ 16 & \mathrm{H} & -3.100849 & 2.313870 & -0.515188 & 1 & 5 & & & \\ 17 & \mathrm{H} & -2.092840 & 2.549611 & 0.970732 & 1 & 5 & & & \\ 18 & \mathrm{H} & 0.602654 & -1.565763 & 1.392641 & 1 & 6 & & & \\ 19 & \mathrm{H} & 1.148715 & -2.914775 & 0.378697 & 1 & 6 & & & \\ 20 & \mathrm{H} & 3.077215 & -1.460468 & 0.838675 & 1 & 7 & & & \\ 21 & \mathrm{H} & 2.712690 & -1.488418 & -0.887803 & 1 & 7 & & & \\ 22 & \mathrm{H} & 3.590142 & 1.960581 & -0.554296 & 1 & 9 & & & \\ 23 & \mathrm{H} & 3.012095 & 0.580034 & 2.062244 & 1 & 10 & & & \\ 24 & \mathrm{H} & -1.588463 & 3.258095 & -0.603660 & 1 & 5 & & & \\ 25 & \mathrm{H} & 2.192003 & 2.010782 & 1.406251 & 1 & 10 & & & \\ 26 & \mathrm{H} & 1.236465 & 0.589220 & 1.906590 & 1 & 10 & & & \\ 27 & \mathrm{H} & 4.445616 & 0.525669 & 0.047571 & 1 & 9 & & & \\ 28 & \mathrm{Mg} & 0.574546 & 0.826185 & -1.034818 & 1 & 11 & 8 & 3 & \end{array}$




\section{Geometry 30}

28

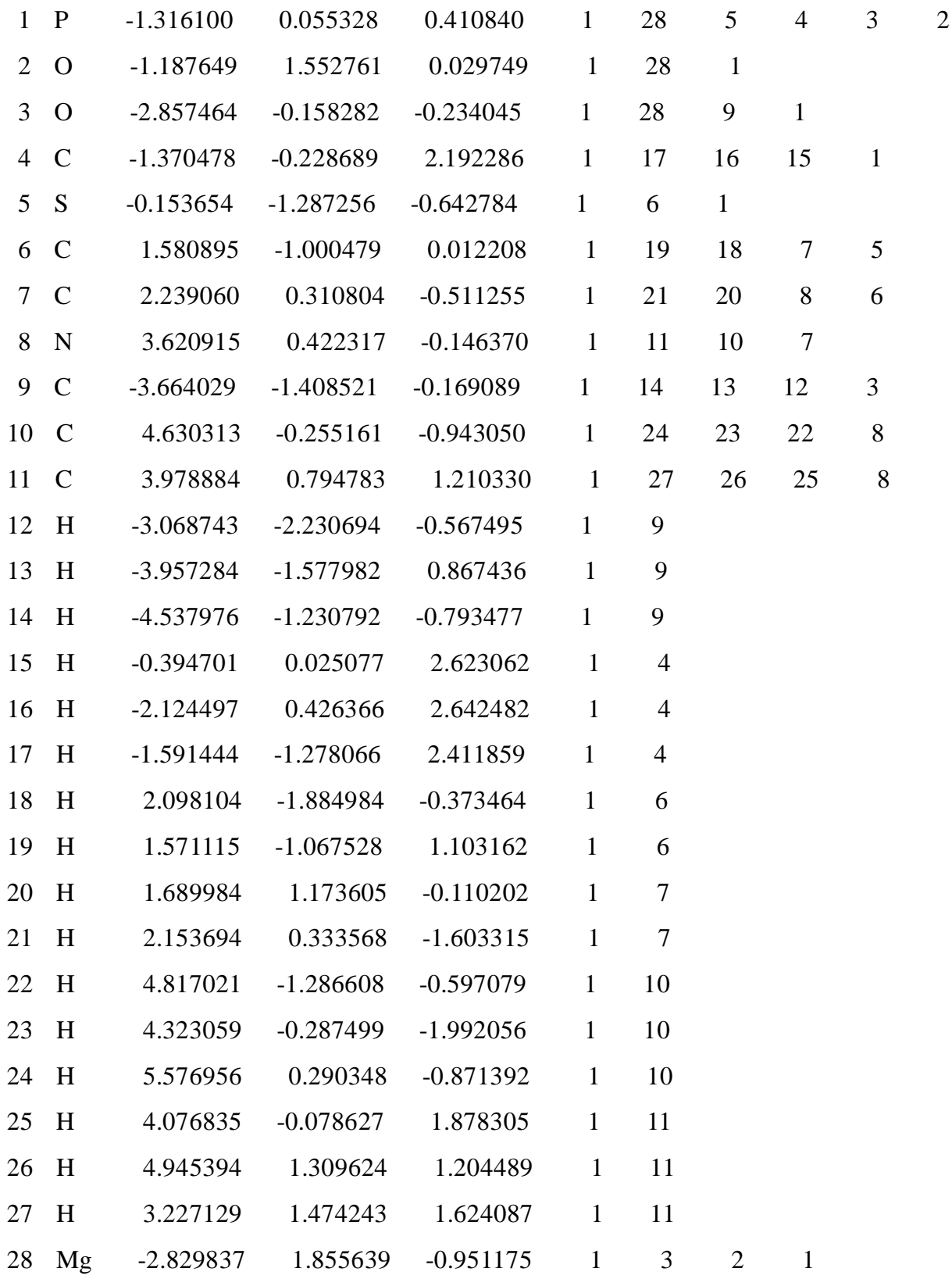




\section{Geometry 31}

28

$\begin{array}{rrrrrrrrrr}1 & \mathrm{P} & 1.770449 & -0.066822 & 0.421494 & 1 & 5 & 4 & 3 & 2 \\ 2 & \mathrm{O} & 0.886881 & -1.153814 & 1.037830 & 1 & 28 & 1 & & \\ 3 & \mathrm{O} & 2.913543 & -0.728154 & -0.441365 & 1 & 9 & 1 & & \\ 4 & \mathrm{C} & 2.430241 & 1.120325 & 1.607883 & 1 & 17 & 16 & 15 & 1 \\ 5 & \mathrm{~S} & 0.514326 & 0.851313 & -1.071145 & 1 & 6 & 1 & & \\ 6 & \mathrm{C} & -0.774582 & 1.783633 & -0.078881 & 1 & 19 & 18 & 7 & 5 \\ 7 & \mathrm{C} & -2.199264 & 1.401819 & -0.499566 & 1 & 21 & 20 & 8 & 6 \\ 8 & \mathrm{~N} & -2.691584 & 0.078518 & 0.009331 & 1 & 28 & 11 & 10 & 7 \\ 9 & \mathrm{C} & 4.175917 & -0.127513 & -0.901072 & 1 & 14 & 13 & 12 & 3 \\ 10 & \mathrm{C} & -3.934863 & -0.274982 & -0.748485 & 1 & 24 & 23 & 22 & 8 \\ 11 & \mathrm{C} & -3.043611 & 0.183186 & 1.459812 & 1 & 27 & 26 & 25 & 8 \\ 12 & \mathrm{H} & 3.964655 & 0.717834 & -1.559122 & 1 & 9 & & & \\ 13 & \mathrm{H} & 4.772606 & 0.171224 & -0.037802 & 1 & 9 & & & \\ 14 & \mathrm{H} & 4.676715 & -0.923168 & -1.449576 & 1 & 9 & & & \\ 15 & \mathrm{H} & 1.622513 & 1.567493 & 2.194573 & 1 & 4 & & & \\ 16 & \mathrm{H} & 3.095738 & 0.580675 & 2.293049 & 1 & 4 & & & \\ 17 & \mathrm{H} & 2.989543 & 1.910726 & 1.097492 & 1 & 4 & & & \\ 18 & \mathrm{H} & -0.609064 & 2.841665 & -0.296198 & 1 & 6 & & & \\ 19 & \mathrm{H} & -0.602118 & 1.639260 & 0.990993 & 1 & 6 & & & \\ 20 & \mathrm{H} & -2.266841 & 1.390064 & -1.592424 & 1 & 7 & & & \\ 21 & \mathrm{H} & -2.882126 & 2.191437 & -0.152377 & 1 & 7 & & & \\ 22 & \mathrm{H} & -4.701707 & 0.502572 & -0.637295 & 1 & 10 & & & \\ 23 & \mathrm{H} & -3.710740 & -0.384539 & -1.814522 & 1 & 10 & & & \\ 24 & \mathrm{H} & -4.352389 & -1.212457 & -0.366506 & 1 & 10 & & & \\ 25 & \mathrm{H} & -3.800391 & 0.960452 & 1.629764 & 1 & 11 & & & \\ 26 & \mathrm{H} & -3.460032 & -0.767321 & 1.811019 & 1 & 11 & & & \\ 27 & \mathrm{H} & -2.162834 & 0.424281 & 2.063111 & 1 & 11 & & & \\ 28 & \mathrm{Ca} & -0.942241 & -1.712079 & -0.161673 & 1 & 8 & 2 & & \\ & & & & & & & & \end{array}$




\section{Geometry 32}

28

$\begin{array}{rrrrrrrrrr}1 & \mathrm{P} & -1.744483 & -0.469765 & 0.319061 & 1 & 11 & 4 & 3 & 2 \\ 2 & \mathrm{O} & -1.262059 & -0.683429 & 1.706211 & 1 & 1 & & & \\ 3 & \mathrm{O} & -1.613886 & 1.171962 & -0.142845 & 1 & 28 & 5 & 1 & \\ 4 & \mathrm{C} & -3.403713 & -1.004822 & -0.151199 & 1 & 15 & 14 & 13 & 1 \\ 5 & \mathrm{C} & -2.599951 & 2.117807 & 0.440819 & 1 & 24 & 17 & 16 & 3 \\ 6 & \mathrm{C} & 0.968393 & -1.963455 & -0.056037 & 1 & 19 & 18 & 11 & 7 \\ 7 & \mathrm{C} & 2.328240 & -1.272800 & -0.167553 & 1 & 21 & 20 & 8 & 6 \\ 8 & \mathrm{~N} & 2.381304 & 0.157992 & 0.273780 & 1 & 28 & 10 & 9 & 7 \\ 9 & \mathrm{C} & 3.749998 & 0.682276 & -0.031033 & 1 & 27 & 22 & 12 & 8 \\ 10 & \mathrm{C} & 2.150497 & 0.261262 & 1.750311 & 1 & 26 & 25 & 23 & 8 \\ 11 & \mathrm{~S} & -0.345774 & -1.211588 & -1.163410 & 1 & 28 & 6 & 1 & \\ 12 & \mathrm{H} & 3.951946 & 0.622197 & -1.106101 & 1 & 9 & & & \\ 13 & \mathrm{H} & -3.624788 & -0.743814 & -1.190771 & 1 & 4 & & & \\ 14 & \mathrm{H} & -3.450429 & -2.092754 & -0.029292 & 1 & 4 & & & \\ 15 & \mathrm{H} & -4.139324 & -0.553649 & 0.523632 & 1 & 4 & & & \\ 16 & \mathrm{H} & -3.582210 & 1.914993 & 0.012473 & 1 & 5 & & & \\ 17 & \mathrm{H} & -2.602539 & 2.015119 & 1.527547 & 1 & 5 & & & \\ 18 & \mathrm{H} & 0.565125 & -1.971275 & 0.959445 & 1 & 6 & & & \\ 19 & \mathrm{H} & 1.065331 & -2.997747 & -0.395124 & 1 & 6 & & & \\ 20 & \mathrm{H} & 3.036098 & -1.862913 & 0.435523 & 1 & 7 & & & \\ 21 & \mathrm{H} & 2.685375 & -1.318217 & -1.202646 & 1 & 7 & & & \\ 22 & \mathrm{H} & 3.833819 & 1.723486 & 0.298192 & 1 & 9 & & & \\ 23 & \mathrm{H} & 2.875262 & -0.352455 & 2.302043 & 1 & 10 & & & \\ 24 & \mathrm{H} & -2.279619 & 3.119865 & 0.153133 & 1 & 5 & & & \\ 25 & \mathrm{H} & 2.286181 & 1.300476 & 2.069158 & 1 & 10 & & & \\ 26 & \mathrm{H} & 1.141129 & -0.064272 & 2.014949 & 1 & 10 & & & \\ 27 & \mathrm{H} & 4.523201 & 0.104564 & 0.492130 & 1 & 9 & & & \\ 28 & \mathrm{Ca} & 0.629636 & 1.482623 & -0.908544 & 1 & 11 & 8 & 3 & \end{array}$




\section{Geometry 33}

28

$\begin{array}{rrrrrrrrrr}1 & \mathrm{P} & -1.838362 & -0.042372 & 0.402686 & 1 & 5 & 4 & 3 & 2 \\ 2 & \mathrm{O} & -0.871695 & 1.165422 & 0.247407 & 1 & 28 & 1 & & \\ 3 & \mathrm{O} & -3.161445 & 0.058149 & -0.452938 & 1 & 9 & 1 & & \\ 4 & \mathrm{C} & -2.459840 & -0.157199 & 2.090258 & 1 & 17 & 16 & 15 & 1 \\ 5 & \mathrm{~S} & -0.879133 & -1.805063 & -0.227698 & 1 & 6 & 1 & & \\ 6 & \mathrm{C} & 0.812994 & -1.599578 & 0.499436 & 1 & 19 & 18 & 7 & 5 \\ 7 & \mathrm{C} & 1.817713 & -0.980072 & -0.486807 & 1 & 21 & 20 & 8 & 6 \\ 8 & \mathrm{~N} & 3.128670 & -0.758385 & 0.065084 & 1 & 11 & 10 & 7 & \\ 9 & \mathrm{C} & -3.320871 & 0.095718 & -1.904187 & 1 & 14 & 13 & 12 & 3 \\ 10 & \mathrm{C} & 4.197720 & -0.696009 & -0.935392 & 1 & 24 & 23 & 22 & 8 \\ 11 & \mathrm{C} & 3.186144 & 0.267458 & 1.044262 & 1 & 27 & 26 & 25 & 8 \\ 12 & \mathrm{H} & -2.924700 & 1.037520 & -2.290103 & 1 & 9 & & & \\ 13 & \mathrm{H} & -2.824162 & -0.762500 & -2.360699 & 1 & 9 & & & \\ 14 & \mathrm{H} & -4.395824 & 0.044439 & -2.069856 & 1 & 9 & & & \\ 15 & \mathrm{H} & -1.633714 & -0.296836 & 2.794034 & 1 & 4 & & & \\ 16 & \mathrm{H} & -2.998373 & 0.765026 & 2.333997 & 1 & 4 & & & \\ 17 & \mathrm{H} & -3.144447 & -1.007103 & 2.165746 & 1 & 4 & & & \\ 18 & \mathrm{H} & 1.134209 & -2.616025 & 0.744696 & 1 & 6 & & & \\ 19 & \mathrm{H} & 0.741891 & -1.052791 & 1.442070 & 1 & 6 & & & \\ 20 & \mathrm{H} & 1.407756 & 0.004452 & -0.882559 & 1 & 7 & & & \\ 21 & \mathrm{H} & 1.897309 & -1.620681 & -1.368943 & 1 & 7 & & & \\ 22 & \mathrm{H} & 4.176719 & -1.593579 & -1.557163 & 1 & 10 & & & \\ 23 & \mathrm{H} & 4.089107 & 0.190861 & -1.590365 & 1 & 10 & & & \\ 24 & \mathrm{H} & 5.167574 & -0.644631 & -0.436091 & 1 & 10 & & & \\ 25 & \mathrm{H} & 2.538562 & 0.080603 & 1.903649 & 1 & 11 & & & \\ 26 & \mathrm{H} & 4.203295 & 0.447012 & 1.398019 & 1 & 11 & & & \\ 27 & \mathrm{H} & 2.852853 & 1.288433 & 0.582141 & 1 & 28 & 11 & & \\ 28 & \mathrm{Ca} & 0.815739 & 2.459531 & -0.193122 & 1 & 27 & 2 & & \\ & & & & & & & & \end{array}$




\section{Geometry 34}

28

$\begin{array}{rrrrrrrrrr}1 & \mathrm{P} & 1.618762 & -0.327750 & -0.457806 & 1 & 5 & 4 & 3 & 2 \\ 2 & \mathrm{O} & 0.863162 & 0.623931 & -1.395127 & 1 & 28 & 1 & & \\ 3 & \mathrm{O} & 1.660853 & 0.688969 & 0.896981 & 1 & 28 & 9 & 1 & \\ 4 & \mathrm{C} & 3.315370 & -0.732501 & -0.903411 & 1 & 17 & 16 & 15 & 1 \\ 5 & \mathrm{~S} & 0.613516 & -2.085226 & -0.029606 & 1 & 6 & 1 & & \\ 6 & \mathrm{C} & -1.140649 & -1.564170 & -0.389101 & 1 & 19 & 18 & 7 & 5 \\ 7 & \mathrm{C} & -1.775352 & -0.659512 & 0.696495 & 1 & 21 & 20 & 8 & 6 \\ 8 & \mathrm{~N} & -2.989890 & 0.012903 & 0.321546 & 1 & 11 & 10 & 7 & \\ 9 & \mathrm{C} & 2.333350 & 0.306385 & 2.155518 & 1 & 14 & 13 & 12 & 3 \\ 10 & \mathrm{C} & -4.254827 & -0.734775 & 0.378312 & 1 & 24 & 23 & 22 & 8 \\ 11 & \mathrm{C} & -2.872499 & 1.058537 & -0.645051 & 1 & 27 & 26 & 25 & 8 \\ 12 & \mathrm{H} & 1.948928 & -0.658545 & 2.491840 & 1 & 9 & & & \\ 13 & \mathrm{H} & 3.411205 & 0.273389 & 1.988851 & 1 & 9 & & & \\ 14 & \mathrm{H} & 2.090519 & 1.080938 & 2.882364 & 1 & 9 & & & \\ 15 & \mathrm{H} & 3.303321 & -1.209739 & -1.890051 & 1 & 4 & & & \\ 16 & \mathrm{H} & 3.914559 & 0.182899 & -0.952100 & 1 & 4 & & & \\ 17 & \mathrm{H} & 3.757182 & -1.429632 & -0.182839 & 1 & 4 & & & \\ 18 & \mathrm{H} & -1.665947 & -2.522352 & -0.450286 & 1 & 6 & & & \\ 19 & \mathrm{H} & -1.151911 & -1.113474 & -1.383189 & 1 & 6 & & & \\ 20 & \mathrm{H} & -1.037637 & 0.120781 & 0.997681 & 1 & 7 & & & \\ 21 & \mathrm{H} & -1.954155 & -1.247351 & 1.602988 & 1 & 7 & & & \\ 22 & \mathrm{H} & -4.398143 & -1.410716 & -0.476664 & 1 & 10 & & & \\ 23 & \mathrm{H} & -4.278714 & -1.320943 & 1.301265 & 1 & 10 & & & \\ 24 & \mathrm{H} & -5.089187 & -0.028815 & 0.407760 & 1 & 10 & & & \\ 25 & \mathrm{H} & -2.379052 & 0.798592 & -1.596752 & 1 & 11 & & & \\ 26 & \mathrm{H} & -3.851137 & 1.483681 & -0.881695 & 1 & 11 & & & \\ 27 & \mathrm{H} & -2.301293 & 1.933940 & -0.185260 & 1 & 28 & 11 & & \\ 28 & \mathrm{Ca} & 0.134427 & 2.335496 & -0.117768 & 1 & 27 & 3 & 2 & \\ & & & & & & & & \end{array}$




\section{Geometry 35}

28

$\begin{array}{rrrrrrrrrr}1 & \mathrm{P} & -1.685212 & -0.769691 & 0.495950 & 1 & 5 & 4 & 3 & 2 \\ 2 & \mathrm{O} & -2.899240 & 0.136741 & 0.188275 & 1 & 28 & 1 & & \\ 3 & \mathrm{O} & -1.588650 & -2.098192 & -0.345299 & 1 & 9 & 1 & & \\ 4 & \mathrm{C} & -1.589064 & -1.349513 & 2.196651 & 1 & 17 & 16 & 15 & 1 \\ 5 & \mathrm{~S} & -0.137415 & 0.613595 & -0.003309 & 1 & 28 & 6 & 1 & \\ 6 & \mathrm{C} & 1.645861 & -0.102180 & 0.190722 & 1 & 19 & 18 & 7 & 5 \\ 7 & \mathrm{C} & 2.698611 & 0.909363 & -0.316769 & 1 & 21 & 20 & 8 & 6 \\ 8 & \mathrm{~N} & 4.018134 & 0.365702 & -0.179860 & 1 & 11 & 10 & 7 & \\ 9 & \mathrm{C} & -1.868676 & -2.279692 & -1.769942 & 1 & 14 & 13 & 12 & 3 \\ 10 & \mathrm{C} & 4.477152 & -0.631271 & -1.139442 & 1 & 24 & 23 & 22 & 8 \\ 11 & \mathrm{C} & 4.637810 & 0.329258 & 1.139488 & 1 & 27 & 26 & 25 & 8 \\ 12 & \mathrm{H} & -2.910966 & -2.024569 & -1.968240 & 1 & 9 & & & \\ 13 & \mathrm{H} & -1.181707 & -1.670644 & -2.362647 & 1 & 9 & & & \\ 14 & \mathrm{H} & -1.694146 & -3.337941 & -1.957190 & 1 & 9 & & & \\ 15 & \mathrm{H} & -1.557199 & -0.499843 & 2.884062 & 1 & 4 & & & \\ 16 & \mathrm{H} & -2.482498 & -1.952716 & 2.396187 & 1 & 4 & & & \\ 17 & \mathrm{H} & -0.702338 & -1.975409 & 2.335425 & 1 & 4 & & & \\ 18 & \mathrm{H} & 1.639295 & -1.025004 & -0.392805 & 1 & 6 & & & \\ 19 & \mathrm{H} & 1.754736 & -0.314279 & 1.255854 & 1 & 6 & & & \\ 20 & \mathrm{H} & 2.626596 & 1.839105 & 0.261130 & 1 & 7 & & & \\ 21 & \mathrm{H} & 2.508012 & 1.142923 & -1.371439 & 1 & 7 & & & \\ 22 & \mathrm{H} & 4.206321 & -1.665267 & -0.869089 & 1 & 10 & & & \\ 23 & \mathrm{H} & 4.071060 & -0.406702 & -2.131125 & 1 & 10 & & & \\ 24 & \mathrm{H} & 5.569124 & -0.584407 & -1.204687 & 1 & 10 & & & \\ 25 & \mathrm{H} & 4.394203 & -0.576506 & 1.718976 & 1 & 11 & & & \\ 26 & \mathrm{H} & 5.725941 & 0.361073 & 1.021785 & 1 & 11 & & & \\ 27 & \mathrm{H} & 4.335850 & 1.209521 & 1.716205 & 1 & 11 & & & \\ 28 & \mathrm{Ca} & -2.552972 & 2.254220 & -0.400387 & 1 & 5 & 2 & & \end{array}$




\section{MP2/6-31+G* OPTIMIZED GEOMETRIES}

\section{Geometry 1}

27

\begin{tabular}{|c|c|c|c|c|c|c|c|c|}
\hline $\mathrm{P}$ & -1.580088 & -0.629152 & 0.422679 & 1 & 5 & 4 & 3 & 2 \\
\hline 0 & -0.861050 & 0.115369 & 1.511326 & 1 & 1 & & & \\
\hline 0 & -2.868073 & 0.115714 & -0.236750 & 1 & 9 & 1 & & \\
\hline $\mathrm{C}$ & -2.349513 & -2.185025 & 0.902533 & 1 & 17 & 16 & 15 & 1 \\
\hline$S$ & -0.332347 & -1.022570 & -1.227304 & 1 & 6 & 1 & & \\
\hline $\mathrm{C}$ & 1.284757 & -0.592757 & -0.480486 & 1 & 19 & 18 & 7 & 5 \\
\hline C & 1.557626 & 0.914621 & -0.567523 & 1 & 21 & 20 & 8 & 6 \\
\hline $\mathrm{N}$ & 2.805825 & 1.366893 & 0.033844 & 1 & 11 & 10 & 7 & \\
\hline $\mathrm{C}$ & -2.730791 & 1.515821 & -0.588490 & 1 & 14 & 13 & 12 & 3 \\
\hline $\mathrm{C}$ & 3.999204 & 0.839186 & -0.611526 & 1 & 24 & 23 & 22 & $\varepsilon$ \\
\hline $\mathrm{C}$ & 2.851168 & 1.196469 & 1.481935 & 1 & 27 & 26 & 25 & 8 \\
\hline $\mathrm{H}$ & -2.476713 & 2.100374 & 0.298101 & 1 & 9 & & & \\
\hline $\mathrm{H}$ & -1.963082 & 1.635731 & -1.357968 & 1 & 9 & & & \\
\hline $\mathrm{H}$ & -3.702729 & 1.814652 & -0.978176 & 1 & 9 & & & \\
\hline $\mathrm{H}$ & -1.580032 & -2.855977 & 1.293168 & 1 & 4 & & & \\
\hline $\mathrm{H}$ & -3.087333 & -1.982756 & 1.684258 & 1 & 4 & & & \\
\hline $\mathrm{H}$ & -2.839604 & -2.651062 & 0.043880 & 1 & 4 & & & \\
\hline $\mathrm{H}$ & 2.019877 & -1.171253 & -1.048975 & 1 & 6 & & & \\
\hline $\mathrm{H}$ & 1.297879 & -0.934991 & 0.556737 & 1 & 6 & & & \\
\hline $\mathrm{H}$ & 0.740525 & 1.443951 & -0.066924 & 1 & 7 & & & \\
\hline $\mathrm{H}$ & 1.559939 & 1.217262 & -1.623145 & 1 & 7 & & & \\
\hline $\mathrm{H}$ & 4.167482 & -0.237543 & -0.424378 & 1 & 10 & & & \\
\hline $\mathrm{H}$ & 3.929048 & 0.998868 & -1.692418 & 1 & 10 & & & \\
\hline $\mathrm{H}$ & 4.874649 & 1.381614 & -0.239685 & 1 & 10 & & & \\
\hline $\mathrm{H}$ & 2.989783 & 0.147068 & 1.798859 & 1 & 11 & & & \\
\hline $\mathrm{H}$ & 3.687152 & 1.780185 & 1.881562 & 1 & 11 & & & \\
\hline $\mathrm{H}$ & 1.919517 & 1.565497 & 1.919612 & 1 & 11 & & & \\
\hline
\end{tabular}

\section{Geometry 2}

28

$\begin{array}{rrrrrrrrrr}1 & \mathrm{P} & 1.227562 & 0.238949 & 0.532012 & 1 & 11 & 4 & 3 & 2 \\ 2 & 0 & 0.447716 & -0.901631 & 1.161042 & 1 & 28 & 1 & \\ 3 & 0 & 1.976164 & -0.168170 & -0.843368 & 1 & 5 & 1 & \end{array}$




\begin{tabular}{|c|c|c|c|c|c|c|c|c|}
\hline 4 & $\mathrm{C}$ & 2.415132 & 1.024609 & 1.626341 & 1 & 15 & 14 & 13 \\
\hline 5 & $\mathrm{C}$ & 2.871910 & -1.325260 & -0.854845 & 1 & 24 & 17 & 16 \\
\hline 6 & $\mathrm{C}$ & -0.924470 & 0.938756 & -1.476882 & 1 & 19 & 18 & 11 \\
\hline 7 & $\mathrm{C}$ & -1.429848 & -0.485117 & -1.244148 & 1 & 21 & 20 & 8 \\
\hline 8 & $\mathrm{~N}$ & -2.046132 & -0.739265 & 0.100307 & 1 & 28 & 10 & 9 \\
\hline 9 & $\mathrm{C}$ & -2.494188 & -2.164155 & 0.199846 & 1 & 27 & 22 & 12 \\
\hline 10 & $\mathrm{C}$ & -3.152312 & 0.202946 & 0.437475 & 1 & 26 & 25 & 23 \\
\hline 11 & $S$ & -0.019219 & 1.783423 & -0.121427 & 1 & 6 & 1 & \\
\hline 12 & $\mathrm{H}$ & -1.636292 & -2.808328 & 0.004900 & 1 & 9 & & \\
\hline 13 & $\mathrm{H}$ & 2.983119 & 1.791433 & 1.093153 & 1 & 4 & & \\
\hline 14 & $\mathrm{H}$ & 1.893805 & 1.468844 & 2.479326 & 1 & 4 & & \\
\hline 15 & $\mathrm{H}$ & 3.097884 & 0.258815 & 2.008877 & 1 & 4 & & \\
\hline 16 & $\mathrm{H}$ & 3.783971 & -1.091362 & -0.301894 & 1 & 5 & & \\
\hline 17 & $\mathrm{H}$ & 2.369496 & -2.194647 & -0.426222 & 1 & 5 & & \\
\hline 18 & $\mathrm{H}$ & -1.754007 & 1.617539 & -1.696082 & 1 & 6 & & \\
\hline 19 & $\mathrm{H}$ & -0.283275 & 0.908816 & -2.362581 & 1 & 6 & & \\
\hline 20 & $\mathrm{H}$ & -2.188519 & -0.720942 & -1.999441 & 1 & 7 & & \\
\hline 21 & $\mathrm{H}$ & -0.612916 & -1.203971 & -1.334851 & 1 & 7 & & \\
\hline 22 & $\mathrm{H}$ & -2.864923 & -2.340523 & 1.210134 & 1 & 9 & & \\
\hline 23 & $\mathrm{H}$ & -3.922292 & 0.130100 & -0.333943 & 1 & 10 & & \\
\hline 24 & $\mathrm{H}$ & 3.106989 & -1.492554 & -1.903804 & 1 & 5 & & \\
\hline 25 & $\mathrm{H}$ & -3.567163 & -0.084032 & 1.404494 & 1 & 10 & & \\
\hline 26 & $\mathrm{H}$ & -2.758111 & 1.216254 & 0.497991 & 1 & 10 & & \\
\hline 27 & $\mathrm{H}$ & -3.286358 & -2.341433 & -0.529955 & 1 & 9 & & \\
\hline 28 & $\mathrm{H}$ & -1.252799 & -0.650430 & 0.782275 & 1 & 2 & 8 & \\
\hline
\end{tabular}

\section{Geometry 5}

28

$\begin{array}{lllllllllll}1 & \mathrm{P} & 1.403826 & -0.088702 & 0.578410 & 1 & 28 & 5 & 4 & 3 & 2 \\ 2 & \mathrm{O} & 0.410312 & -0.905052 & 1.385000 & 1 & 28 & 1 & & \\ 3 & \mathrm{O} & 2.637496 & -0.984141 & 0.096419 & 1 & 9 & 1 & & \\ 4 & \mathrm{C} & 2.029218 & 1.335971 & 1.477074 & 1 & 17 & 16 & 15 & 1 \\ 5 & \mathrm{~S} & 0.529347 & 0.483525 & -1.236241 & 1 & 6 & 1 & & \\ 6 & \mathrm{C} & -0.939315 & 1.430295 & -0.651990 & 1 & 19 & 18 & 7 & 5 \\ 7 & \mathrm{C} & -2.249403 & 0.728602 & -1.000398 & 1 & 21 & 20 & 8 & 6 \\ 8 & \mathrm{~N} & -2.559294 & -0.445890 & -0.153741 & 1 & 28 & 11 & 10 & 7\end{array}$




$\begin{array}{rrrrrrrrrr}9 & \mathrm{C} & 3.867894 & -0.437248 & -0.470684 & 1 & 14 & 13 & 12 & 3 \\ 10 & \mathrm{C} & -3.615642 & -1.244426 & -0.820790 & 1 & 24 & 23 & 22 & 8 \\ 11 & \mathrm{C} & -3.063067 & -0.002320 & 1.168795 & 1 & 27 & 26 & 25 & 8 \\ 12 & \mathrm{H} & 3.638658 & 0.245096 & -1.292207 & 1 & 9 & & & \\ 13 & \mathrm{H} & 4.440429 & 0.063885 & 0.311111 & 1 & 9 & & \\ 14 & \mathrm{H} & 4.414332 & -1.301729 & -0.840374 & 1 & 9 & & \\ 15 & \mathrm{H} & 1.197941 & 1.971403 & 1.795072 & 1 & 4 & & \\ 16 & \mathrm{H} & 2.545422 & 0.973448 & 2.372882 & 1 & 4 & & \\ 17 & \mathrm{H} & 2.716292 & 1.927210 & 0.866515 & 1 & 4 & & \\ 18 & \mathrm{H} & -0.902294 & 2.395366 & -1.165857 & 1 & 6 & & \\ 19 & \mathrm{H} & -0.860370 & 1.630094 & 0.420517 & 1 & 6 & & \\ 20 & \mathrm{H} & -2.211363 & 0.394276 & -2.042571 & 1 & 7 & & \\ 21 & \mathrm{H} & -3.068990 & 1.463240 & -0.923396 & 1 & 7 & & \\ 22 & \mathrm{H} & -4.518077 & -0.643006 & -1.002612 & 1 & 10 & \\ 23 & \mathrm{H} & -3.243185 & -1.620215 & -1.777331 & 1 & 10 & \\ 24 & \mathrm{H} & -3.890286 & -2.090997 & -0.185386 & 1 & 10 & & \\ 25 & \mathrm{H} & -3.969025 & 0.611693 & 1.061972 & 1 & 11 & & \\ 26 & \mathrm{H} & -3.308696 & -0.877388 & 1.777589 & 1 & 11 & & \end{array}$

Geometry 11

28

$\begin{array}{rrrrrrrrrr}1 & \mathrm{P} & -1.728094 & -0.240560 & 0.368026 & 1 & 11 & 4 & 3 & 2 \\ 2 & \mathrm{O} & -1.029748 & 0.696162 & 1.338188 & 1 & 28 & 1 & & \\ 3 & \mathrm{O} & -3.050049 & 0.331400 & -0.337533 & 1 & 5 & 1 & & \\ 4 & \mathrm{C} & -2.322403 & -1.772724 & 1.082413 & 1 & 15 & 14 & 13 & 1 \\ 5 & \mathrm{C} & -3.155506 & 1.720665 & -0.759877 & 1 & 24 & 17 & 16 & 3 \\ 6 & \mathrm{C} & 0.904662 & -1.548105 & -0.417221 & 1 & 19 & 18 & 11 & 7 \\ 7 & \mathrm{C} & 2.275448 & -0.968190 & -0.758645 & 1 & 21 & 20 & 8 & 6 \\ 8 & \mathrm{~N} & 2.631885 & 0.227340 & 0.033122 & 1 & 28 & 10 & 9 & 7 \\ 9 & \mathrm{C} & 3.736140 & 0.936883 & -0.648771 & 1 & 27 & 22 & 12 & 8 \\ 10 & \mathrm{C} & 3.091344 & -0.178159 & 1.379704 & 1 & 26 & 25 & 23 & 8 \\ 11 & \mathrm{~S} & -0.459705 & -0.640917 & -1.263147 & 1 & 6 & 1 & & \\ 12 & \mathrm{H} & 3.399799 & 1.285630 & -1.628864 & 1 & 9 & & & \\ 13 & \mathrm{H} & -2.852683 & -2.355327 & 0.325287 & 1 & 4 & & & \end{array}$




$\begin{array}{rrrrrrr}14 & \mathrm{H} & -1.491819 & -2.357027 & 1.487033 & 1 & 4 \\ 15 & \mathrm{H} & -3.006245 & -1.520942 & 1.899137 & 1 & 4 \\ 16 & \mathrm{H} & -2.956414 & 2.381553 & 0.085530 & 1 & 5 \\ 17 & \mathrm{H} & -2.468293 & 1.913854 & -1.587392 & 1 & 5 \\ 18 & \mathrm{H} & 0.740737 & -1.550489 & 0.664584 & 1 & 6 \\ 19 & \mathrm{H} & 0.850026 & -2.584151 & -0.763340 & 1 & 6 \\ 20 & \mathrm{H} & 3.034978 & -1.754383 & -0.605238 & 1 & 7 \\ 21 & \mathrm{H} & 2.304523 & -0.699364 & -1.820014 & 1 & 7 \\ 22 & \mathrm{H} & 4.044251 & 1.798797 & -0.049711 & 1 & 9 \\ 23 & \mathrm{H} & 3.971023 & -0.837449 & 1.323194 & 1 & 10 \\ 24 & \mathrm{H} & -4.183208 & 1.836817 & -1.097125 & 1 & 5 \\ 25 & \mathrm{H} & 3.367151 & 0.710117 & 1.956347 & 1 & 10 \\ 26 & \mathrm{H} & 2.296428 & -0.702732 & 1.916375 & 1 & 10 \\ 27 & \mathrm{H} & 4.613053 & 0.286232 & -0.787560 & 1 & 9 \\ 28 & \mathrm{Na} & 0.786405 & 1.732532 & 0.533547 & 1 & 8\end{array}$

\section{Geometry 19}

28

$\begin{array}{rrrrrrrrrr}1 & \mathrm{P} & -1.561659 & -0.175243 & 0.497749 & 1 & 5 & 4 & 3 & 2 \\ 2 & \mathrm{O} & -0.606705 & 0.437475 & 1.488288 & 1 & 28 & 1 & & \\ 3 & \mathrm{O} & -2.356728 & 1.004667 & -0.287343 & 1 & 9 & 1 & & \\ 4 & \mathrm{C} & -2.770798 & -1.296797 & 1.212307 & 1 & 17 & 16 & 15 & 1 \\ 5 & \mathrm{~S} & -0.546022 & -1.088438 & -1.087004 & 1 & 6 & 1 & & \\ 6 & \mathrm{C} & 0.793009 & -1.950075 & -0.168200 & 1 & 19 & 18 & 7 & 5 \\ 7 & \mathrm{C} & 2.173552 & -1.433055 & -0.557620 & 1 & 21 & 20 & 8 & 6 \\ 8 & \mathrm{~N} & 2.493947 & -0.089299 & -0.057199 & 1 & 28 & 11 & 10 & 7 \\ 9 & \mathrm{C} & -3.512263 & 0.758070 & -1.141423 & 1 & 14 & 13 & 12 & 3 \\ 10 & \mathrm{C} & 3.732476 & 0.368478 & -0.710503 & 1 & 24 & 23 & 22 & 8 \\ 11 & \mathrm{C} & 2.697407 & -0.114725 & 1.401966 & 1 & 27 & 26 & 25 & 8 \\ 12 & \mathrm{H} & -3.271883 & 0.018491 & -1.909213 & 1 & 9 & & & \\ 13 & \mathrm{H} & -4.357841 & 0.429679 & -0.534834 & 1 & 9 & & & \\ 14 & \mathrm{H} & -3.739797 & 1.716245 & -1.604030 & 1 & 9 & & & \\ 15 & \mathrm{H} & -2.240058 & -2.103287 & 1.727478 & 1 & 4 & & & \\ 16 & \mathrm{H} & -3.367154 & -0.747778 & 1.948577 & 1 & 4 & & & \\ 17 & \mathrm{H} & -3.420700 & -1.733746 & 0.450015 & 1 & 4 & & & \\ 18 & \mathrm{H} & 0.721362 & -3.009331 & -0.430960 & 1 & 6 & & & \end{array}$




$\begin{array}{llllccc}19 & \mathrm{H} & 0.619164 & -1.855255 & 0.906442 & 1 & 6 \\ 20 & \mathrm{H} & 2.257224 & -1.416185 & -1.650082 & 1 & 7 \\ 21 & \mathrm{H} & 2.917672 & -2.164107 & -0.187568 & 1 & 7 \\ 22 & \mathrm{H} & 4.583390 & -0.302514 & -0.507887 & 1 & 10 \\ 23 & \mathrm{H} & 3.581253 & 0.421151 & -1.792402 & 1 & 10 \\ 24 & \mathrm{H} & 3.997491 & 1.364016 & -0.339627 & 1 & 10 \\ 25 & \mathrm{H} & 3.459902 & -0.854704 & 1.696604 & 1 & 11 \\ 26 & \mathrm{H} & 3.046551 & 0.868943 & 1.734506 & 1 & 11 \\ 27 & \mathrm{H} & 1.759065 & -0.333608 & 1.914652 & 1 & 11 \\ 28 & \mathrm{~K} & 0.693956 & 2.149591 & -0.048535 & 1 & 8\end{array}$

\section{Geometry 26}

28

\begin{tabular}{|c|c|c|c|c|c|c|c|c|c|}
\hline 1 & $\mathrm{P}$ & 1.472922 & -0.262131 & 0.253883 & 1 & 5 & 4 & 3 & 2 \\
\hline 2 & $\mathrm{O}$ & 0.364495 & -1.332575 & 0.552004 & 1 & 28 & 1 & & \\
\hline 3 & $\mathrm{O}$ & 2.480191 & -0.814162 & -0.794046 & 1 & 9 & 1 & & \\
\hline 4 & $\mathrm{C}$ & 2.213013 & 0.430046 & 1.718325 & 1 & 17 & 16 & 15 & 1 \\
\hline 5 & $S$ & 0.202964 & 1.073892 & -0.877022 & 1 & 28 & 6 & 1 & \\
\hline 6 & $\mathrm{C}$ & -1.041604 & 1.733637 & 0.357912 & 1 & 19 & 18 & 7 & 5 \\
\hline 7 & $\mathrm{C}$ & -2.406289 & 1.158241 & -0.026488 & 1 & 21 & 20 & 8 & 6 \\
\hline 8 & $\mathrm{~N}$ & -2.383662 & -0.352324 & 0.017235 & 1 & 28 & 11 & 10 & 7 \\
\hline 9 & $\mathrm{C}$ & 3.869860 & -0.359535 & -1.116152 & 1 & 14 & 13 & 12 & 3 \\
\hline 10 & $\mathrm{C}$ & -3.344726 & -0.918440 & -0.998299 & 1 & 24 & 23 & 22 & \\
\hline 11 & $\mathrm{C}$ & -2.775267 & -0.845579 & 1.389087 & 1 & 27 & 26 & 25 & $\varepsilon$ \\
\hline 12 & $\mathrm{H}$ & 3.814862 & 0.637053 & -1.551693 & 1 & 9 & & & \\
\hline 13 & $\mathrm{H}$ & 4.466347 & -0.391985 & -0.206065 & 1 & 9 & & & \\
\hline 14 & $\mathrm{H}$ & 4.212965 & -1.092954 & -1.840386 & 1 & 9 & & & \\
\hline 15 & $\mathrm{H}$ & 1.444021 & 0.800550 & 2.402909 & 1 & 4 & & & \\
\hline 16 & $\mathrm{H}$ & 2.766494 & -0.373885 & 2.223227 & 1 & 4 & & & \\
\hline 17 & $\mathrm{H}$ & 2.900125 & 1.243408 & 1.461033 & 1 & 4 & & & \\
\hline 18 & $\mathrm{H}$ & -1.029074 & 2.823526 & 0.257874 & 1 & 6 & & & \\
\hline 19 & $\mathrm{H}$ & -0.743926 & 1.482323 & 1.381657 & 1 & 6 & & & \\
\hline 20 & $\mathrm{H}$ & -2.667263 & 1.465231 & -1.044587 & 1 & 7 & & & \\
\hline 21 & $\mathrm{H}$ & -3.179704 & 1.536000 & 0.653302 & 1 & 7 & & & \\
\hline 22 & $\mathrm{H}$ & -4.348286 & -0.528993 & -0.803474 & 1 & 10 & & & \\
\hline 23 & $\mathrm{H}$ & -3.029443 & -0.629046 & -2.003770 & 1 & 10 & & & \\
\hline
\end{tabular}




\begin{tabular}{|c|c|c|c|c|c|c|}
\hline 24 & -3.356543 & -2.006900 & -0.908854 & 1 & 10 & \\
\hline 25 & -3.819993 & -0.583473 & 1.578554 & 1 & 11 & \\
\hline $\mathrm{H}$ & -2.656043 & -1.930221 & 1.429194 & 1 & 11 & \\
\hline 27 & -2.138582 & -0.385170 & 2.147788 & 1 & 11 & \\
\hline $\mathrm{Be}$ & -0.784746 & -0.716457 & -0.270804 & 1 & 8 & 5 \\
\hline
\end{tabular}

\section{Geometry 28}

28

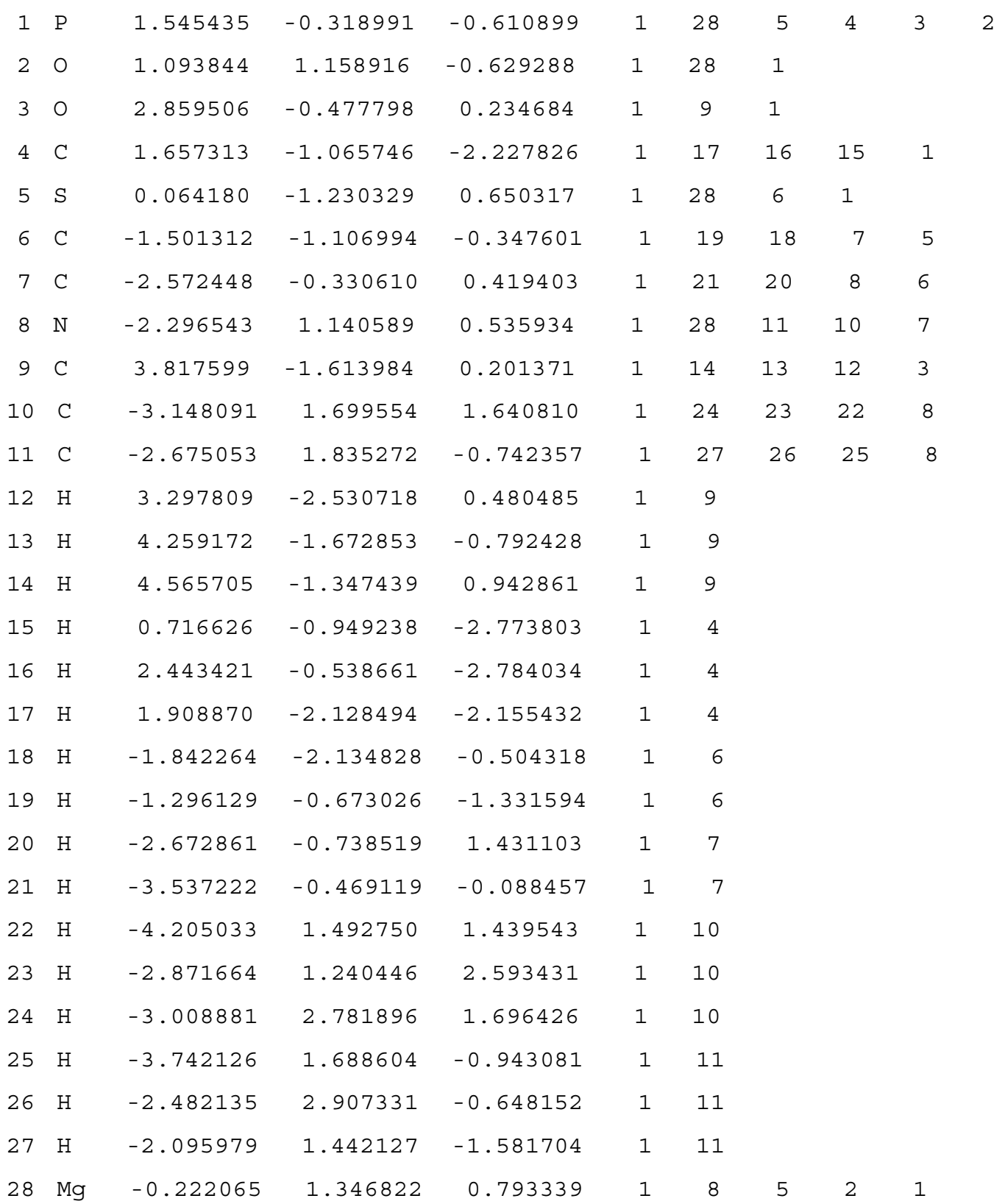




\section{Geometry 31}

28

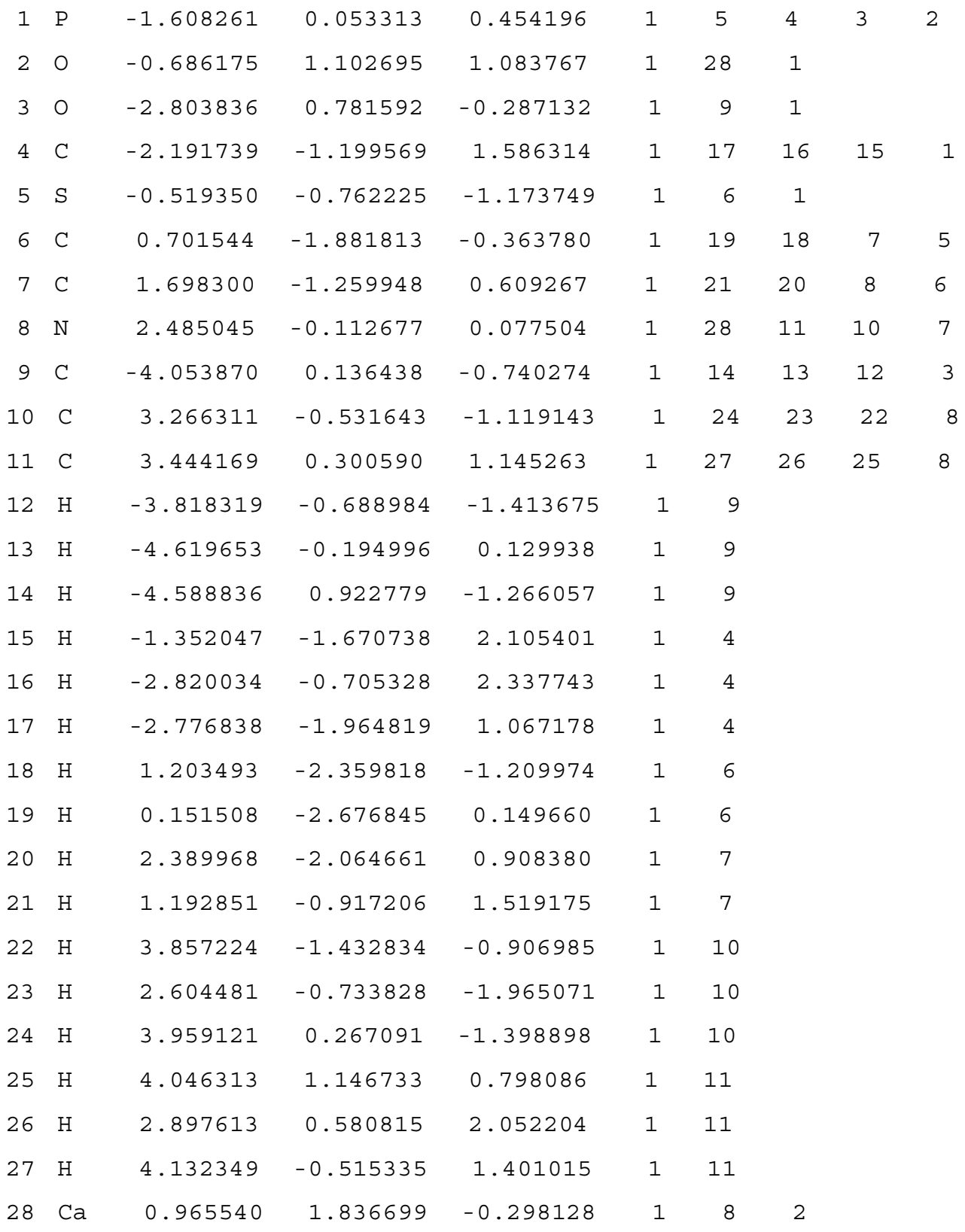

